\title{
Nonlinear Measures for Characterizing Rough Surface Morphologies
}

\author{
Jané Kondev 1 \\ Institute for Advanced Study, Olden Lane, Princeton, NJ 08540, and \\ Department of Physics, Princeton University, Princeton, NJ 08540 \\ Christopher L. Henley and David G. Salinasl \\ Laboratory of Atomic and Solid State Physics, Cornell University, Ithaca, New York, 14853
}

(September 1, 2018)

\begin{abstract}
We develop a new approach to characterizing the morphology of rough surfaces based on the analysis of the scaling properties of contour loops, i.e. loops of constant height. Given a height profile of the surface we perform independent measurements of the fractal dimension of contour loops, and the exponent that characterizes their size distribution. Scaling formulas are derived and used to relate these two geometrical exponents to the roughness exponent of a self-affine surface, thus providing independent measurements of this important quantity. Furthermore, we define the scale dependent curvature and demonstrate that by measuring its third moment departures of the height fluctuations from Gaussian behavior can be ascertained. These nonlinear measures are used to characterize the morphology of computer generated Gaussian rough surfaces, surfaces obtained in numerical simulations of a simple growth model, and surfaces observed by scanning-tunnelingmicroscopes. For experimentally realized surfaces the self-affine scaling is cut off by a correlation length, and we generalize our theory of contour loops to take this into account.
\end{abstract}

\section{INTRODUCTION}

Random surfaces are widely used in the physical sciences to model phenomena ranging from the extremely small (quantum gravity) to the very large (Earth's relief). They describe crack fronts in materials science [3], ripple-wave turbulence [4], passive tracers in two-dimensional fluid flows [5.67, cloud perimeters [7],8], shapes of stromatolites (conjectured fossil accretions of ancient bacteria) [9], to mention but a few recent examples. Our focus in this paper is on the morphology of deposited metal films, which develop random self-affine surfaces under several quite different non-equilibrium growth conditions, as indicated by theoretical, numerical, and experimental results over the past decade 10,11.

Surface configurations are parametrized by a two-dimensional field $h(\mathbf{x})$ which represents the height of the surface above a reference plane $\{\mathbf{x}\}$. Theoretically the dynamics of a growing surface are described by a continuum (Langevin) equation giving $d h(\mathbf{x}) / d t$ as a sum of a Gaussian white noise term, to mimic the random deposition of atoms, and a polynomial of various gradients of $h(\mathbf{x})$, to model relaxation processes on a coarse grained scale. The nonequilibrium growth behavior is due to the interplay of the deposition and relaxation terms.

Different relaxation terms are appropriate for different growth conditions, and the notion of universality has been taken over from the study of critical phenomena. Namely, it is believed that there are only a few distinct universality classes of growth characterized by exponents which describe the temporal and spatial scaling of the growing interface.

One would guess that even a snapshot of the morphology should carry the evidence of the non-equilibrium, nonlinear growth process which produced it, and should differ measurably from surfaces produced in equilibrium or by a linear process, even if they share the same scaling exponents.

This motivates a search for roughness measures independent of the quadratic ones (e.g. the rms height), which might identify important distinctions between different surface models that have similar spatial power spectra. Such measures, although motivated in the context of self-affine or multi-affine surfaces, should be handy even for surfaces showing no self-affine regime. They can quantify features of morphology which are presently characterized by eye, which should permit a more systematic comparison between observations and models than at present. One can imagine that, armed with two or three kinds of roughness measures tuned to different qualitative aspects of the surfaces morphology, one could construct empirical "phase diagrams" in this two or three-dimensional parameter space, e.g. mapping out domains in the parameter space that correspond to various growth conditions.

Given the surface as parametrized by an array of heights - obtained, e.g., from a simulation or a scanning-tunnelingmicroscope (STM) experiment [12] - we ask, in what different ways can the surface morphology be characterized? In general, one requires more than one characterization to confirm a match between experimental and simulation data, or to convincingly verify self-affineness. For the applied problems of growing flat surfaces (e.g. for semiconductor 
devices) or regularly modulated ones (e.g. to nanofabricate arrays of quantum dots), it is also desirable to develop independent measures that quantify different aspects of a rough surface's geometry. In this paper, we propose two categories of novel measure for characterizing spatial correlations of rough surfaces. These measures are usable on any kind of rough model, and require no dynamical information, so they should be useful in analyzing not only experiments on solid films, but all the diverse phenomena mentioned above.

\section{A. Outline of the paper}

We start with a short review (Section III) of self-affine geometry in terms of its real-space, Fourier space and fractal properties. In section III we introduce the non-linear measures, the scale dependent curvature and the loop measures, for which various scaling relations are derived in section IV. These scaling relations are modified in the presence of a (time dependent) cutoff length-scale above which the height fluctuations are no longer self-affine, and this situation is described towards the end of section IV. This concludes the first half of the paper which deals with the theory of non-linear measures.

The second half of the paper is devoted to analyzing data obtained in numerical simulations and experiments using the measures introduced in the first part. It starts off with section $\mathrm{V}$ where we present the results of our simulations of random Gaussian surfaces for various values of the roughness exponent $0 \leq \alpha \leq 1$. These simulations serve to confirm the various scaling relations derived earlier. In section $\mathrm{VI}$ the non-linear measures are applied to a non-equilibrium growth model, the so-called single step model, which is known to belong to the KPZ universality class. Finally, in section VII we demonstrate the usefulness of our measures for analyzing experimental data on the example provided by an STM image of a growth roughened metal film. The discussion section (section VIII) summarizes our main results, gives a critical comparison between the newly introduces measures and those used previously, and points out some interesting new directions in which progress can be made. The three appendices are reserved for details of the calculation of the loop correlation exponent in the case of equilibrium rough surfaces (appendix A), details of the derivation of percolation exponents for contours of uncorrelated heights (appendix B), as well as a full description of the loop finding algorithm which is at the heart of the numerical simulations and the loop analysis of STM data (appendix G).

\section{SELF-AFFINE GEOMETRY}

Here we review the scaling properties of self-affine interfaces in real-space and in Fourier-space, as well as the fractal geometry of their level sets. The surface is fully specified by the height field $h(\mathbf{x})$, which may be the microscopic heights of individual surface atoms above the substrate, as measured by an STM, or it may be a coarse grained quantity representing the average of individual atomic heights over a region 13 ].

The defining property of self-affine surfaces is their invariance under rescaling. Namely, the probability distribution function $(\mathrm{PDF})$ for $h(\mathbf{x})$ is such that

$$
h(\mathbf{x}) \cong b^{-\alpha} h(b \mathbf{x})
$$

for any $b>1$, where $\alpha$ is the roughness exponent; here the symbol $\cong$ means "statistically equivalent with respect to the PDF". In other words, if we stretch the surface by a rescale factor $b$ in the horizontal direction (= parallel to the reference plane $\mathbf{x}$ ), then to obtain a statistically equivalent surface, we must stretch by a factor $b^{\alpha}$ in the vertical direction (= the perpendicular direction of the heights $h(\mathbf{x}))$. A central theme of this paper is the different ways of determining $\alpha$, given a height profile $h(\mathbf{x})$.

A self-affine surface is rough if $\alpha>0$. Furthermore, for the surface to exhibit a two-dimensional character (at distances much larger than the surface width) the rescale factor in the vertical direction $\left(b^{\alpha}\right)$ can not exceed the one in the horizontal direction (b), i.e., we require $\alpha \leq 1$.

\section{A. Real-space properties}

The self-affine scaling of the height is typically measured by the height-correlation function

$$
D_{2}(\mathbf{r})=\left\langle[h(\mathbf{x}+\mathbf{r})-h(\mathbf{x})]^{2}\right\rangle \sim|\mathbf{r}|^{2 \alpha},
$$

where the scaling with separation $|\mathbf{r}|$ is a direct consequence of the self-affine property, Eq. (2.1), which states that $h$ has a scaling dimension $\alpha$. 
In experiments the correlations that lead to self-affine scaling of the surface develop over time, which we take to be measured from the start of the deposition process. Namely, after time $t$ self-affine scaling will be observed only up-to length scales smaller then the correlation length $\xi(t)$. Physically the height correlations develop due to the various surface relaxation processes that are present under the given growth conditions.

Numerical simulations of various surface growth models, as well as experiments under different conditions, have shown that $\xi$ grows with the duration of the deposition process, $t$, according to the dynamical scaling relation [14]:

$$
\xi(t) \sim t^{1 / z} .
$$

It is believed that there are only a few different universality classes of growth each characterized by the exponents $\alpha$ and $z$ [11,14]. Experimental efforts have been focused on extracting these exponents from data obtained using various surface-sensitive methods: X-rays or helium diffraction, STM scans, etc. [10] In this paper, we will be almost entirely concerned with the spatial (equal-time) correlations.

\section{B. Fourier-space properties}

The power spectrum of a self-affine surface

$$
S(\mathbf{q})=\left\langle|\tilde{h}(\mathbf{q})|^{2}\right\rangle
$$

is defined in terms of the Fourier transformed height

$$
\tilde{h}(\mathbf{q})=\int d^{2} \mathbf{x} h(\mathbf{x}) e^{-\mathbf{i q} \cdot \mathbf{x}} .
$$

The height correlation function is linearly related to the height power spectrum,

$$
D_{2}(\mathbf{r})=\int d^{2} \mathbf{q} S(\mathbf{q})\left(e^{\mathrm{iq} \cdot \mathbf{r}}-1\right),
$$

as is any other translation-invariant expectation quadratic in heights, such as the net variance. Eq. (2.6) and Eq. (2.2) imply the scaling

$$
S(\mathbf{q}) \sim|\mathbf{q}|^{-2(1+\alpha)},
$$

for small values of $|\mathbf{q}|$. In the case of a surface with a finite correlation length, $S(\mathbf{q})$ crosses over to a constant value for $|\mathbf{q}|<1 / \xi(t)$; this situation is discussed in more detail in Sec. IVE.

Clearly, $S(\mathbf{q})$ does not uniquely characterize a self-affine ensemble of surfaces. For example, it is invariant under $h(\mathbf{x}) \rightarrow-h(\mathbf{x})$, yet surfaces produced in non-equilibrium growth typically break the up/down symmetry. Furthermore, given any $S(\mathbf{q})$ one can always construct a Gaussian ensemble by linear addition of Fourier components - we do this in Sec. $\mathrm{V}$ - yet the real growth process is typically nonlinear, and the surface is non-Gaussian. Indeed, confirmation of the scaling given by Eqs. 2.2 or 2.7 in experiments can not be interpreted as conclusive evidence for a self-affine geometry: that is a property of the whole ensemble and so requires proper scaling of all moments and correlations, not just the second moment.

\section{Quadratic roughness measures}

The quantities (2.2) and (2.4) are quadratic measures of roughness, which we shall also call "linear"; untill recently, no other kind was in use.

The height-correlation function, Eq. (2.2), is the most standard measure in theoretical discussions, in that "roughness" is defined by the divergence of this function as its argument $\mathbf{r}$ approaches infinity. (Non-monotonic behavior of this function has also been used [15] to measure the characteristic spatial scale of mounds or other patterns in non-self-affine surfaces.)

On the other hand, the Fourier power spectrum (2.4) is central in theoretical derivations but rarely used in experimental analysis (except for Ref. [16]). This seems to be the best quadratic measure, in that it most cleanly separates the contributions from fluctuations on different length scales, and it shows the sharpest knee (in a log-log plot) where self-affine scaling is cut off. 
Another quadratic measure is the total variance of $h(\mathbf{x})$ in a in a box of size $b$, as a function of $b$ [17, 18]:

$$
\left\langle\left(h(\mathbf{x})-\bar{h}_{b}\right)^{2}\right\rangle_{b},
$$

where $\bar{h}_{b} \equiv\langle h(\mathbf{x})\rangle_{b}$, and $\langle\ldots\rangle_{b}$ means the spatial average is only taken over a square of side $b$ centered on $\mathbf{x}_{0}$; this variance should be averaged over different choices of $\mathbf{x}_{0}$.

\section{Fractal properties}

Self-affine surfaces are fractals only in a generalized sense, since the horizontal direction rescales differently from the vertical direction. On the other hand, the level set of such a surface (defined as its intersection with a horizontal plane) is a fractal object 19]; see Fig. 1, below. Different planes of intersection give statistically equivalent level sets, since the height fluctuations of a rough surface are unbounded. Level sets consist of contour loops which are the connected components. We expect these to be fractal as well, with a fractal dimension smaller then the dimension of the whole level set, which is simply the union of all contour loops of the same height. Furthermore, contour loops come in all sizes limited only by the system size, and an exponent can be defined that characterizes their size distribution. Since contour loops are connected clusters their geometrical exponents are analogous to those defined for percolation clusters.

We will show that the scaling of contour loops uniquely specifies the scaling of the associated self-affine rough surface; this will be expressed in formulas giving the geometrical exponents in terms of the roughness exponent $\alpha$. It is somewhat surprising that, by doing measurements solely on the level set, information can be obtained about the out-of-plane fluctuations of the surface. For experiments that yield only level-set data without the heights, (e.g. freeze-fracture electron microscopy [20]) our contour-loop analysis is the only route to extracting the roughness exponent.

\section{NONLINEAR MEASURES}

In the past the analysis of rough surfaces mostly relied on measures which probed the second moment of the heights, such as the height-correlation function or the power spectrum (defined in Eq. (2.2) and Eq. (2.7) below). But that is inherently insufficient to distinguish different growth ensembles or even to verify self-affineness.

Therefore, to more fully characterize rough surfaces we introduce in this section two new types of non-linear measures, i. e., measures that are not linearly related to the structure function of the height field. Non-linear measures of the first type (Sec. III A) are moments of the "scale-dependent curvature", a modification of the standard height correlation function which can identify deviations from Gaussianness of the height fluctuations, in particular the skew (up/down asymmetry) at various length scales. (We will compare these to existing non-quadratic roughness measures in the Discussion part of Sec. VIII.)

Measures of the second type were introduced in Ref. [21]; they are distributions of three different geometrical quantities defined for contour-loops (or simply "loops") of constant height, which make up the level sets of the height function. These measures are associated with geometrical exponents that characterize contour loops on self-affine rough surfaces: the loop correlation exponent, the fractal dimension of a loop, and the length distribution exponent.

\section{A. Scale-dependent curvature}

The obvious real-space-based nonquadratic generalization of the height-correlation function is

$$
\left\langle\left[(h(\mathbf{x}+\mathbf{r})-h(\mathbf{x})]^{3}\right\rangle ;\right.
$$

however, this is identically zero on an isotropic surface (and whenever $\mathbf{r} \rightarrow-\mathbf{r}$ is a symmetry). To escape this problem, we observe that $h(\mathbf{x}+\mathbf{r})-h(\mathbf{x})$ is a sort of first difference at scale $r$, and replace it by a sort of second difference. Namely, we define the "curvature at $\mathbf{x}$ on scale $b$ " as

$$
C_{b}(\mathbf{x})=\sum_{m=1}^{M}\left[h\left(\mathbf{x}+b \mathbf{e}_{m}\right)-h(\mathbf{x})\right]
$$


where the offset directions $\left\{\mathbf{e}_{m}\right\}$ are a fixed set of vectors summing to zero. In our numerical implementation of this measure, where $\{\mathbf{x}\}$ is a square lattice, we choose four such offsets related by $90^{\circ}$ rotations, pointing either along the $\{10\}$ or the $\{11\}$ type directions. Those two sets of offsets should give equivalent results (for the same $b$ ), provided the surface is statistically invariant under rotations in the reference plane. We then define curvature moments $\left\langle C_{b}^{q}\right\rangle$ for integer powers $q$.

The first moment of $C_{b}$ is manifestly zero; the second moment is linearly related to the height-correlation function:

$$
\left\langle C_{b}(\mathbf{x})^{2}\right\rangle=M \sum_{m=1}^{M} D_{2}\left(b \mathbf{e}_{m}\right)-\frac{1}{2} \sum_{m, n=1}^{M} D_{2}\left(b\left(\mathbf{e}_{m}-\mathbf{e}_{n}\right)\right) .
$$

(This is shown by inserting Eq. (3.2) and then decoupling each term of the double sum using the identity $\left(h_{m}-\right.$ $\left.\left.h_{0}\right)\left(h_{n}-h_{0}\right)=\frac{1}{2}\left\{\left(h_{m}-h_{0}\right)^{2}+\left(h_{n}-h_{0}\right)^{2}-\left(h_{m}-h_{n}\right)^{2}\right\}.\right)$

The higher moments of $C_{b}$ serve to measure the (possible) deviation of the height fluctuations from the Gaussian distribution. For example, if the surface has up/down symmetry $h \leftrightarrow-h$ (as all Gaussian surfaces do), $\left\langle\left[C_{b}(\mathbf{x})\right]^{3}\right\rangle$ vanishes. On the other hand, non-equilibrium grown surfaces often have rounded "hilltops" and sharp "valleys"; that tends to make $\left\langle C_{b}^{3}\right\rangle>0$, a signature of "skew" in the distribution. Similarly, the fourth moment can also be used to test whether the surface is Gaussian, since in that case

$$
\left\langle\left[C_{b}(\mathbf{x})\right]^{4}\right\rangle /\left\langle\left[C_{b}(\mathbf{x})\right]^{2}\right\rangle^{2}=3 .
$$

For a self-affine surface

$$
\left\langle\left[C_{b}(\mathbf{x})\right]^{q}\right\rangle \simeq \mathrm{const}|\mathbf{x}|^{q \alpha}
$$

follows from Eq. (2.1); of course the coefficient might be zero as is the case for odd $q$, when the height field has up/down symmetry.

Functions such as $\left\langle C_{b}^{q}\right\rangle$ (as a function of $b$ ) or $D_{q}(r)$ (as a function of $r$ ) can also be used as "spectra" of the height fluctuations for non self-affine surfaces. That is, differences in the behavior of the function in different ranges of $b$ or $r$ reveal qualitative differences of the surface morphology on the corresponding length scales.

In principle, $q$-th order moments may scale with well-defined exponents $\alpha_{q}$, yet the surface is not self-affine since $\alpha_{q} \neq q \alpha$ violating Eq. (3.5); this is called a "multifractal" or, more precisely, "multiaffine" surface [22]. Slow transients of multiaffine behavior (up to $\sim 10^{8}$ steps in $d=1+1$ and $\sim 10^{3}$ steps in $d=2+1$ ) have been seen recently in numerical simulations of growth models 23 (which, however, are believed to be asymptotically self-affine). The analogous higher order structure functions are a central issue in turbulence, where the violation of self-affine (Kolmogorov) scaling is well established and is associated with intermittency of the velocity field fluctuations [24].

The scale dependent curvature can be contrasted with Krug's height-difference moments [22],

$$
D_{q}(\mathbf{r}) \equiv\left\langle|h(\mathbf{x}+\mathbf{r})-h(\mathbf{x})|^{q}\right\rangle,
$$

a natural generalization of the height-correlation function using an absolute value to avoid the trivial cancellation in Eq. (3.1). Das Sarma and collaborators [25.23] used (3.6) to test for multi-affine behavior (whereby the $1 / q$ power of the $q$ moment scales with exponent depending on $q$, unlike the simpler self-affine case). For odd $q$, Eq. (3.6) is insensitive to the up-down symmetry (or lack thereof) since it is nonzero anyhow. Our "curvature" seems to be the simplest function that detects the skew locally.

\section{B. Fractal dimension of contour loops}

For the remainder of this section, we must define the loop ensemble. Consider a contour plot of a rough surface with a fixed spacing $\Delta$ between heights of successive level sets. We take it to be an arbitrary constant much smaller than the typical (r.m.s.) fluctuation of $h(\mathbf{x})$. The value of $\Delta$ does not affect our exponents and we need to consider it explicitly only in the arguments of Sec. IV A; in other places we may implicitly scale $h(\mathbf{x})$ such that $\Delta=1$. In STM images of rough metal surfaces $\Delta$ is usually the height of a single step on the surface.

The contour plot consists of closed nonintersecting lines in the plane that connect points of equal height, which we call contour loops (see Fig.1, below). Every random-surface configuration maps to a configuration of contour-loops; when the probability weights of the respective configurations are taken into account, this defines a mapping of the random-surface ensemble, to the contour-loop ensemble. The contour loop ensemble arising from self-affine random surfaces is (we shall argue) self-similar; the loops are connected clusters that can be studied using scaling, just as (critical) percolation clusters have been analyzed in previous work [26]. 
For every contour loop in the loop ensemble we define a loop length $s$ and a loop radius $R$. In all the examples we study the heights are defined on an $L \times L$ square lattice with lattice constant $a$. The loop length is measured with a ruler of length $a$ while the loop radius (really a diameter) is defined as the side of the smallest box that completely covers the loop; see Fig. 18 .

In the loop ensemble we define a joint distribution $\tilde{n}(s, R)$ (independent of the contour spacing $\Delta$ ) such that the number of loops with length in $(s, s+d s)$ and radius in $(R, R+d R)$, per unit area, is

$$
\Delta^{-1} \tilde{n}(s, R) d s d R .
$$

The factor $\Delta^{-1}$ has the obvious significance that if one halves the contour spacing, one has twice as many contours.

Assuming that the loop ensemble is scale invariant, we expect that $\tilde{n}(s, R)$ has a scaling form

$$
\tilde{n}(s, R) \sim s^{-y} f_{n}\left(s / R^{D_{f}}\right) .
$$

Here $D_{f}$ is the fractal dimension, and $y$ is simply related to the length distribution exponent $\tau$, which we define in the next section.

In practice the exponent $D_{f}$ is measured by the scaling relation

$$
\langle s\rangle(R) \sim R^{D_{f}},
$$

where

$$
\langle s\rangle(R) \equiv \int_{R}^{R+\delta R} s \tilde{n}(s, R) d s d R / \int_{R}^{R+\delta R} \tilde{n}(s, R) d s d R
$$

is the average loop length for loops whose radius falls in the interval $(R, R+\delta R), \delta R \ll R$. The scaling in Eq. (3.9) follows immediately from the assumed scaling form in Eq. (3.8).

The dimension defined in Eq. (3.9) is really the scaling dimension of the loop length, i.e., it defines the relation between bigger and smaller loops in the distribution. On the other hand, the proper fractal dimension (either the Hausdorff dimension $D_{H}$ or the self-similarity dimension) refers to the relation between bigger and smaller pieces of the same loop. Thus $D_{H}$ is defined by $s \sim a^{-D_{H}}$, i.e. how the loop length scales with the ruler size. When the contour-loop distribution is self similar (as we shall assume), the two kinds of dimensions are equivalent.

\section{Loop length distribution exponent}

We define the loop number density $\tilde{P}(s)$ so that $\Delta^{-1} \tilde{P}(s) d s$ is the total number of loops, per unit area (measured in sites), with lengths in $(s, s+d s)$; a related distribution of loop lengths, $P(s)$, is defined such that $\Delta^{-1} P(s) d s$ is the number of loops passing through a fixed point (say the origin) with lengths in the range $(s, s+d s)$. In lattice models (including our numerical examples in Sections $\mathrm{V}$, V1, and $\mathrm{VII}$ ), $s$ is an integer and $P(s)$ is essentially the probability that the loop has length $s$.

From comparison to Eq. (3.7) it is obvious that

$$
\tilde{P}(s)=\int_{0}^{\infty} \tilde{n}(s, R) d R .
$$

Since the total number of sites along a loop is equal to its length $s$ we have

$$
P(s)=s \tilde{P}(s) ;
$$

the additional factor of $s$ is because each site could be the origin in the definition of $P(s)$.

Assuming that the loop ensemble is scale invariant we can define the length distribution exponent $\tau$ by

$$
P(s) \sim s^{-(\tau-1)} ; \quad \tilde{P}(s) \sim s^{-\tau} .
$$

This is to hold for large contour loops, i.e. those of radius much bigger then the microscopic scale $a$. Indeed, inserting Eq. (3.8) into Eq. (3.11) gives Eq. (3.13), with

$$
y=\tau+1 / D_{f} .
$$


On the other hand, we could also define $\tilde{n}(R)$ such that $\Delta^{-1} \tilde{n}(R) d R$ is the total number of loops, per unit area, whose radius is in the range $(R, R+d R)$. Obviously

$$
\tilde{n}(R)=\int_{0}^{\infty} \tilde{n}(s, R) d s .
$$

Doing the integral and then eliminating $y$ using (3.14) gives

$$
\tilde{n}(R) \sim 1 / R^{1+D_{f}(\tau-1)}
$$

We would have obtained the same result more quickly (and more dubiously) had we assumed a strict relationship between radius and length, $s=($ const $) R^{D_{f}}$, rather than write (3.8).

\section{Loop correlation function}

The loop correlation function $G(\mathbf{r})$ measures the probability that two points separated by $\mathbf{r}$ lie on the same contour loop. This correlation function is non-local, for the connectedness of the two points depends on every site on the portion of loop between them. This loop correlation function should be distinguished from the level-set correlation function which simply measures the probability that two points separated by $\mathbf{r}$ are at the same height. For the loop correlation function to be well defined the contour lines are considered to be of finite width given by the microscopic scale $a$. Due to rotational symmetry of the loop ensemble, $G(\mathbf{r})$ depends on $r=|\mathbf{r}|$ only, and for large separations $(r \gg a)$ we expect it to fall off as a power law:

$$
G(r) \sim \frac{1}{r^{2 x_{l}}} .
$$

This equation defines the loop correlation exponent $x_{l}$ which is at the heart of the scaling theory of contour loops developed below.

\section{SCALING RELATIONS}

In this section we derive scaling relations among the roughness exponent $\alpha$, and the three geometrical exponents - $D_{f}, \tau$, and $x_{l}$ - associated with contour-loops and defined in Sec. III. These formulas are corollaries of the selfaffineness of the rough surface, Eq. (2.1). Furthermore, for growth on an initially flat substrate the heights will be uncorrelated beyond a certain time-dependent length scale and the large contour loops are best modeled as hulls of percolation clusters. This implies a crossover to a different set of exponents as worked out in Sec. IVE. The scaling relations - including the finite-size and finite-time forms in Sec. IV D 1 and Sec. IVE 1 - will serve as a useful tool for analyzing the surface morphologies obtained from numerical simulations and in experiments (see sections $\mathrm{VI}$ and VII).

There are three stages of the main derivation. First, we establish a relationship between the self-affine exponent $\alpha$ and the loop-size distribution exponent $\tau$; it is analogous to the hyperscaling relation among percolation exponents. Second, we find a sum rule (analogous to the susceptibility sum rule) relating the loop correlation exponent $x_{l}$ of Section IIID and the loop-size distribution exponent $\tau$. Third, we present a conjecture that the loop correlation exponent has a value $x_{l}=1 / 2$, which is super-universal in the sense that it is independent of $\alpha$. (This conjecture is supported by an exact calculation of $x_{l}$ in the extreme cases, i.e. $\alpha=0$ (equilibrium rough case) and $\alpha=1$.) Finally, these relations taken together yield formulas for $D_{f}$ and $\tau$ (Eq. (4.13)) as a function of $\alpha$.

\section{A. Hyperscaling relation}

If we parametrize a loop as $\mathbf{l}(s)$, where $s$ is the arc length as measured by a ruler of length $a$, then after the rescaling given by Eq. (2.1) it is mapped to,

$$
\mathbf{l}(s) \rightarrow b^{-1} \mathbf{l}\left(b^{D_{f}} s\right) .
$$

This scaling property of the contour ensemble justifies the power law dependence of $G(r)$ on $r$ and $P(s)$ on $s$, in Eq. (3.17) and Eq. (3.13) respectively. 
In writing Eq. (4.1), we made a nontrivial hypothesis that the contours of the height function obtained by coarsegraining a given realization of $h(\mathbf{x})$ are statistically the same as the coarse-grained version of the contours of $h(\mathbf{x})$. We know of no coarse-graining procedure for the height function which assures that the contours will stay the same. It will happen that, near a saddle-point of $h(\mathbf{x})$, two loops (both of height $h_{\text {lev }}$ ) approach closely, but the coarse-graining shifts the height of the saddle-point across $h_{\text {lev }}$ so that the coarse-grained versions of the loops coalesce into one loop. Whether this phenomenon makes a relevant contribution to our scaling relations depends on the frequency of close approaches [27].

To determine the scaling of $\tilde{n}(R)$ first apply the rescaling Eq. (2.1) to each configuration of $h(\mathbf{r})$; this maps the contour ensemble to a new contour ensemble with rescaled contour interval $\Delta^{\prime}=b^{-\alpha} \Delta$. The total number of contours with radii in the range $(R, R+d R)$, in a box of side $L$, is $L^{2} \Delta^{-1} \tilde{n}(R) d R$, by our definition in Sec. III G. Since the contours are mapped 1-to-1 (according to the hypothesis in Eq. (4.1)), we can equate this with the number of new contours in a box of side $L / b$ and of radius in $(R / b, R / b+d R / b)$, which is $(L / b)^{2} \Delta^{\prime-1} \tilde{n}^{\prime}(R / b) d R^{\prime} / b$. On the other hand, by self-affineness the new height ensemble, is statistically identical to the original one (for large $R$ ); this holds as well for the new contour ensemble, thus $\tilde{n}^{\prime}(R) \equiv \tilde{n}(R)$. So we obtain $\tilde{n}(R / b)=b^{3-\alpha} \tilde{n}(R)$, which implies the scaling behavior

$$
\tilde{n}(R) \sim R^{-3+\alpha} .
$$

Equating Eq. (4.2) and Eq. (3.16) leads to the first scaling relation (called "hyperscaling")

$$
D_{f}(\tau-1)=2-\alpha .
$$

This scaling relation has been derived previously by Huber et al. [28] in a slightly different context, and in a somewhat different form by Isichenko and Kalda [29]. Unlike the usual hyperscaling relation for percolation clusters which can be derived from the assumption that the number of large clusters does not grow with scale of observation, here that number grows as a power with exponent $\alpha$ [30].

\section{B. Sum rule}

A second scaling relation can be derived from a sum rule. To start off, let's separately consider the loop correlation function for different loop sizes $s$. Let $G_{s}(\mathbf{r})$ be the probability that point $\mathbf{x}+\mathbf{r}$ is on the same loop as $\mathbf{x}$, given that the loop has length $s$. In light of the self-similarity of the loop ensemble, it is reasonable to assume

$$
G_{s}(\mathbf{r}) \sim s^{m}|\mathbf{r}|^{-a} f_{G s}\left(r / s^{1 / D_{f}}\right),
$$

where $m$ and $a$ are as-yet undetermined exponents, and $f_{G s}()$ is a scaling function. The reason we must scale $r$ by $s^{1 / D_{f}}$ is that this is the typical diameter $R$ of the loop $\left(s \sim R^{D_{f}}\right)$.

Now, the sum of $G_{s}(\mathbf{r})$ over all lattice points is the expectation of the total number of points in the loop, which was given to be $s$, hence (substituting from (4.4)) [31]

$$
s=\int d^{2} \mathbf{r} G_{s}(\mathbf{r}) \sim s^{(2-a) / D_{f}+m}
$$

which gives one relation between the exponents $a$ and $m$ introduced in (4.4):

$$
2-a=D_{f}(1-m)
$$

On the other hand, the total loop correlation is the integral of $G_{s}(\mathbf{r})$ over the loop distribution function $P(s)$ given by Eq. (3.13), thus

$$
G(\mathbf{r})=\int d s P(s) G_{s}(\mathbf{r}) \sim r^{-a}\left(r^{D_{f}}\right)^{m+2-\tau}=r^{D_{f}(3-\tau)-2}
$$

where (4.6) was used to eliminate both $a$ and $m$ in the result. Equating the exponent of $G(\mathbf{r})$ in (4.7) to the one defined by (3.17), we obtain the scaling relation

$$
D_{f}(3-\tau)=2-2 x_{l} .
$$

The above scaling relations, Eq. (4.8) and Eq. (4.3), can be combined into expressions (which were originally presented in Ref. [21]) for the fractal dimension $D_{f}$ and the exponent $\tau$ : 


$$
\begin{gathered}
D_{f}=2-x_{l}-\alpha / 2 \\
\tau-1=\frac{2-\alpha}{2-x_{l}-\alpha / 2} .
\end{gathered}
$$

The first scaling relation is reminiscent of the relation

$$
D=2-\alpha
$$

due to Mandelbrot 19]. The important difference is that Eq. (4.11) gives the fractal dimension $D$ of the level set of a random self-affine surface, and not the fractal dimension of a single contour loop. (We emphasize this point because there has been some confusion in the literature where the two dimensions have been equated.)

Olami and Zeitak [32] considered the same loop ensemble, but mostly focused their attention on the "islands" contained in the loops rather than the contours; their " $\tau$ " exponent (which we call $\tau_{\mathrm{ZO}}$ ) refers to the distribution of island sizes. They derived a formula $\tau_{\mathrm{ZO}}=2-\alpha / 2$ (in our notation). It is easy to show $2\left(\tau_{\mathrm{ZO}}-1\right)=D_{f}(\tau-1)-$ the "2" here is the fractal dimension of these islands [32]; upon inserting this conversion, their formula turns out to say $D_{f}(\tau-1)=2-\alpha$, which is the same as our Eq. (4.10).

\section{Loop correlation exponent}

Now we turn our attention to the contour correlation exponent, and we conjecture that

$$
x_{l}=1 / 2
$$

is super-universal in that it is independent of $\alpha$.

In the case of an $\alpha=0$ Gaussian surface, we know $x_{l}=1 / 2$ exactly for a solvable statistical-mechanics model of contour loops, equivalent to the critical $O(2)$ loop model on the honeycomb lattice [33. Details are in Appendix A. By invoking universality this is valid for all logarithmically rough random Gaussian surfaces.

The exact value of $x_{l}$ can also be determined for $\alpha=1$. Namely, the fractal dimension $\left(D_{f}\right)$ of a contour loop must satisfy $D_{f} \leq D$ since it is a subset of the level set, which has dimension $D=2-\alpha=1$; Eq. (4.11). On the other hand $D_{f} \geq 1$ since a loop has topological dimension one. From these inequalities we conclude that for $\alpha=1$ the fractal dimension of a contour loop is $D_{f}=1$. This in turn leads to $x_{l}=1 / 2$, from Eq. (4.9).

The validity of conjecture (4.12) for general $\alpha$ has been checked, to date, only through the numerical simulations reported in Section $\mathrm{V}$ and in numerical simulations of Zeng et al. [34].

Since $x_{l}=1 / 2$ for $\alpha=0$ and $\alpha=1$, a proof of monotonicity of $x_{l}$ with $\alpha$ would suffice to establish the conjecture. Even that is very difficult owing to the non-local definition of the loop correlation function.

\section{Combined scaling relations}

Equipped with the (super-universal) conjectured value of the loop exponent $x_{l}=1 / 2$, and the scaling relations, Eqs. (4.9) and (4.10), we find the following formulas for the geometrical exponents of contour loops of a self-affine surface with roughness exponent $\alpha$ :

$$
\begin{aligned}
D_{f} & =\frac{3-\alpha}{2} \\
\tau-1 & =\frac{4-2 \alpha}{3-\alpha} .
\end{aligned}
$$

These relations form the basis of the contour loop analysis of rough surfaces, which we implement in the following sections.

Our formula for $D_{f}$ differs from the one proposed by Isichenko [35]

$$
D_{f}^{\text {Isichenko }}=\frac{10-3 \alpha}{7},
$$

which was derived from an approximate "multiscale" analysis. We note that the formula for $D_{f}$ in Eq. (4.14) gives the wrong result in the $\alpha=0$ case, where $D_{f}=3 / 2$ is exact [36]. 


\section{Finite-size scaling}

For realistic rough surfaces the self-affine scaling will be cut off at large lengths either by the correlation length or the system size.

In the case that self-affine scaling is cut off only by the system size $L$, we can extend the power laws derived above for the average loop length, the size distribution of loops, and the loop correlation function, into scaling forms:

$$
\begin{aligned}
\langle s\rangle(R, L) & =R^{D_{f}} f_{s}(R / L) \\
P(s, L) & =s^{-(\tau-1)} f_{P}\left(s / L^{D_{f}}\right) \\
G(\mathbf{r}, L) & =|\mathbf{r}|^{-2 x_{l}} f(|\mathbf{r}| / L) .
\end{aligned}
$$

In the case that the self affine scaling is cut-off by a finite correlation length $\xi(t)<L$, our three contour-loop measures will display crossover effects to a different set of power laws; we turn to this problem next.

\section{E. Percolation crossover}

For a surface roughened by growth self affine scaling is expected to hold only up to a finite correlation length $\xi(t)$ growing with time as Eq. (2.3). At early enough stages of growth (i.e. while $\xi(t)<L$ ) the statistics at scales beyond $\xi(t)$ depend on the initial state. Then the contour loops of the surface will also exhibit crossover behavior where loops whose linear size (as measured by the radius $R$ ) is less than the correlation length will scale according to the formulas derived above, while the large loops will exhibit scaling with percolation exponents.

Say the initial surface is flat (which we assume henceforth). Then it turns out (see Appendix B) that the contour loops at scale $R>\xi(t)$ are boundaries of percolation clusters. Although this new contour loop ensemble corresponds to a non-self-affine surface, it still exhibits scaling and we derive its three loop exponents in terms of known percolation exponents. In some cases, it turns out, the exponent values from the percolation regime and from the self-affine surface are not so different; thus a careless analysis might yield spurious exponents.

It is easy to see that at $r>\xi$, we can model the actual heights (not height differences) as statistically independent, since (by definition of the correlation length) the distance $\xi(t)$ is the farthest that an event can influence another in time $t$.

In appendix B, we derive the geometrical exponents

$$
D_{f, p}=7 / 4=1.75, \tau_{p}=18 / 7=2.571,2 x_{l, p}=5 / 4=1.25
$$

which apply to loops at scales larger than $\xi(t)$ (the percolation-regime scaling). These exponents are the same for any $\alpha$. An important corollary is that power-law scaling in the loop analysis is not necessarily a signature of self-affine behavior. Indeed, most real surfaces never reach a clear self-affine regime, hence their loops are probably in the percolation regime.

\section{Finite-time crossover scaling forms}

The complete crossover between the self-affine and percolation regimes is described by scaling forms parallel to (4.15). First consider the height structure factor. Since, as noted above, the heights are independent, their (spatial) power spectrum is flat in Fourier space: $S(\mathbf{q}) \sim$ const, for $|\mathbf{q}|<1 / \xi(t)$; on the other hand, for $|\mathbf{q}|>1 / \xi(t)$ the surface has already developed a self-affine state so Eq. (2.7) does hold. The two behaviors should be combined via a scaling function $f_{S}()$ :

$$
S(\mathbf{q} ; t)=|\mathbf{q}|^{-2(1+\alpha)} f_{S}\left(\mathbf{q} t^{1 / z}\right)
$$

see Fig. 2(a).

Thus, at times $t$ such that $\xi(t) \ll L$, the behaviors (3.9), (3.13), and (3.17) are generalized to

$$
\begin{aligned}
\langle s\rangle(R ; t) & =R^{D_{f}} f_{s p}(R / \xi(t)) \\
P(s ; t) & =s^{-(\tau-1)} f_{P p}\left(s / \xi(t)^{D_{f}}\right) \\
G(r) & =r^{-2 x_{l}} f_{G p}(r / \xi(t)) .
\end{aligned}
$$


In each case, the scaling function is unity for argument zero, while for argument large it scales as a power law needed to give the correct exponent for the percolation regime, as calculated in Appendix B 2. The $t$ dependence for the prefactor of each percolation-regime power law is given by the requirement to patch the above two dependences together when the scaling-function argument is of order unity.

Figures 2 (b)-(d) illustrate the shapes of the three loop measures. Notice that the "knee" around $r=\xi(t)$ appears more strikingly in the Fourier analysis than in any of the loop analyses. Although the percolation-regime and selfaffine exponents have fairly similar values, the difference grows larger as $\alpha$ gets larger. The crossover is evident in our simulated Gaussian data (see Fig. 10).

\section{SIMULATION: GAUSSIAN RANDOM SURFACES}

Here we test the validity of our scaling relations and the effectiveness of determining $\alpha$ from contour loops, under the controlled circumstances provided by computer generated surfaces with known $\alpha$. The surfaces we construct are self-affine with Gaussian fluctuations of the height.

\section{A. Construction}

Random Gaussian surfaces are generated numerically as an $L / a \times L / a$ matrix $h(\mathbf{x})$ of real-valued heights associated with the vertices $\{\mathbf{x}\}$ of a square lattice of size $L$, with lattice constant $a$. A particular realization of $h(\mathbf{x})$ is given by Fourier transforming $\tilde{h}(\mathbf{q})$ where the wave-vectors $\mathbf{q}$ take their values in the first Brillouin zone $[-\pi / a, \pi / a] \times[\pi / a, \pi / a]$. Each Fourier component $\tilde{h}(\mathbf{q})$ is an independent Gaussian random variable with a q-dependent variance given by

$$
\left\langle|\tilde{h}(\mathbf{q})|^{2}\right\rangle=\frac{1}{\left(\mathbf{q}^{2}\right)^{1+\alpha}} .
$$

For $0 \geq \alpha \geq 1$ surfaces generated in this way are self-affine and rough, with a roughness exponent $\alpha$.

The $\alpha=0$ case of random Gaussian surfaces is familiar as: (i) the equilibrium-rough surface (compare Eq. (A1)), (ii) the surface in the Edwards-Wilkinson model, and (iii) the Coulomb gas representation of a two-dimensional critical model [33] (see appendix A]. The case $\alpha=1$ appears in the Mullins-Herring (diffusive relaxation) model $(\alpha=1)$ of non-equilibrium surface growth [37].

\section{Comparison to other studies}

A popular algorithm for generating self-affine surfaces is "random midpoint displacement, with random successive addition" 38, 18]. This method iterates a step in which, starting with a self-affine surface on a coarse grid of lattice constant $2 a$, one generates heights on a new grid of lattice constant $a$ by interpolation, and then adds to them random increments proportional to $a^{\alpha}$. Such an ensemble need not be Gaussian or have up-down symmetry, but commonly does [18]. We note that (i) the variance of a site's height (relative to the initial flat surface) depends on what iteration that site appeared, i.e. on how many times 2 can be divided into the site coordinates; (ii) height-difference correlations do not always grow with distance (they are smaller between two sites that appeared in early iterations) and they have the anisotropy of the lattice even at large distances. We believe our Fourier construction of self-affine surfaces (Sec. $\mathrm{VA}$ ) is preferable because the resulting ensemble is (i) spatially homogeneous and (ii) isotropic, on scales beyond a couple of lattice constants.

\section{B. Curvature measurements}

We measured moments $\left\langle C_{b}^{m}\right\rangle(m=2,3,4)$ of the scale-dependent curvature, as defined in Sec. III A, for Gaussian surfaces generated by the Fourier method described above. This data (in Fig. 3) is a kind of check on the Fourier method since the mean over an infinite number of samples can be computed analytically.

Self-affine scaling is evident on the log-log plot of the even moments in Fig. 3 (upper plot). The roughness exponent

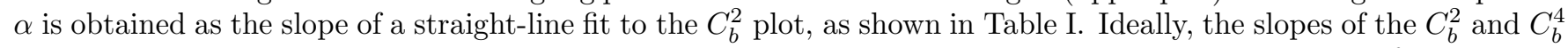
$\log -\log$ plots should be $2 \alpha$ and $4 \alpha$ with exactly the input $\alpha$ values used in constructing the random surfaces. This is spoiled somewhat in practice by discrete-lattice effects for $b \leq 3$ and by finite-size effects when $b>L / 4$. Furthermore, 
$\left\langle C_{b}^{4}\right\rangle /\left\langle C_{b}^{2}\right\rangle^{2}$ should be exactly 3 for every $b$ value, even those for which the power-law dependence on $b$ fails, since this is true for any Gaussian random variable. Indeed, the measured ratio is close to 3 .

The third moment of $C_{b}$ is shown in Fig. 3. Independent of $\alpha,\left\langle C_{b}^{3}\right\rangle$ is roughly zero, as expected for a random Gaussian surface which posses a $h \rightarrow-h$ symmetry (i.e., the valley bottoms and the hill tops are equivalent for a Gaussian surface).

\section{Loop measurements}

The primary motivation for our Gaussian surface simulations was an initial test of the scaling predictions for the contour loop exponents from Sec. IV. A contour plot of a sample surface configuration for $\alpha=0.4$ is shown in Fig. 11. (A similar plot for $\alpha=0$ was published in Ref. 21.)

\section{Measurement procedure}

In a single run, which would typically take 10 minutes on an Sun Sparc5 workstation, 25 surfaces of specified roughness $\alpha$ were generated. For each surface typically 400 points were chosen at random, and through each point a contour loop was constructed using the loop finding algorithm as explained in Appendix C. While each loop was being traced points along the loop were used to evaluate the loop correlation function $G(r)$. For each contour loop its radius and length were measured and used to determine the length distribution of contour loops $(P(s))$ and the average loop length $\langle s\rangle$ as a function of the loop radius $R$.

\section{Results}

In order to measure the geometrical exponents $D_{f}, \tau$, and $x_{l}$ we plotted the data for system size $L=512$ on a $\log -\log$ graph and performed least-squares linear fits. Data was selected for fitting from the range in which a well

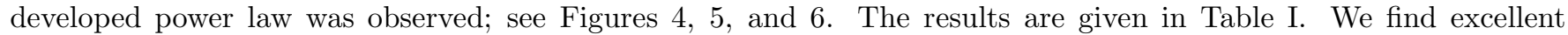
agreement between the predictions of the scaling theory and the measured geometrical exponents. In particular, note that the simulations confirm the super-universal nature of the loop correlation exponent $x_{l}=1 / 2$.

The loop correlation function $G(r)$ has a a size dependence which biases a direct fit to the exponent $2 x_{l}$; finitesize scaling (see below) partially overcomes this systematic error. Our theory (Sec. IV a universal exponent $2 x_{l}=1$; in fact, as shown in Fig. 6, $G(r)$ itself appears practically independent of $\alpha$. Closer examination reveals that the coefficient in $G(r) \sim 1 / r$ decreases slightly as $\alpha$ grows. Furthermore, the fitted values of $2 x_{l}$ (see Table il) decrease a bit with $\alpha$, which we attribute to the systematic error just mentioned, combined with the small $\alpha$-dependence of the shape of the "knee" in the finite-size behavior of Fig. 8. There is no indication in the extracted $2 x_{l}$ values of any non-monotonic dependence on $\alpha$; as shown in section IV , monotonicity of $x_{l}(\alpha)$ is sufficient to prove $2 x_{l}=1$, independent of $\alpha$.

A better measure of the geometrical exponents was obtained from a finite size scaling analysis of the data. Using the scaling forms in Eq. (4.15) we produced data collapses ("scaling plots"). Sample data for the $\alpha=0.4$ case are given in Figs. 1(a) and 8(a); the data collapse is shown in Figs. 7(b) and \&(b). From the loop-size distribution plots like Fig. (7)(b), we extracted both the exponents $D_{f}$ and $\tau-2$. Similarly, we obtained $2 x_{l}$ from the loop correlation function Fig. 8(b); in this case we don't fit another exponent since $r$ obviously scales as $L^{1}$. (We did not carry out finite-size scaling of the $\langle s\rangle$ versus $R$ plots such as Fig. 1 , since there was no obvious change in the slope as a function

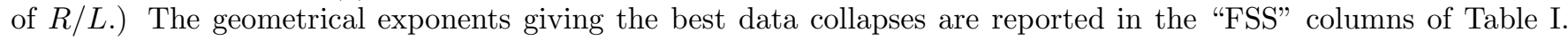
The reported uncertainties were estimated by the interval over which changes in the exponent value did not visibly worsen the data collapse.

Note in Table 1 how the finite-size scaling exponents agree better with the scaling theory of Sec. IV than the exponents obtained from "direct" fitting of the data to power laws. The discrepancy becomes more obvious at larger values of $\alpha$. We infer from this that Gaussian surfaces with a large value of the roughness have more pronounced finite size effects which lead to an overestimate of $D_{f}$ and $\tau$. This is of relevance to experimental data where the system size is typically not a tunable parameter, and the geometrical exponents are necessarily measured using the direct-fit method. 


\section{Relation to a previous simulation}

Numerical measurements of the fractal dimension of contour loops have been done by Avellaneda et al. [39]. They found $D_{f}=1.28 \pm 0.015$ for an $\alpha=0.5$ surface, which is close to the predicted value $D_{f}=1.25$, from Eq. (4.13). They also measured the combination $D_{f}(\tau-2)$ (their " $\alpha$ ") which describes the scaling of the probability that a loop passing through a fixed point has a radius larger than $\rho$, with $\rho$. (We evaluate this quantity by integrating $n(s, R)=s \tilde{n}(s, R)$, from Eq. (3.8), over all $s$ and for $R>\rho$ ). The numerical result they quote, $D_{f}(\tau-2)=0.21 \pm 0.017$, is in fair agreement with our prediction $D_{f}(\tau-2)=(1-\alpha) / 2=0.25$ for $\alpha=0.5$, which follows from Eq. (4.13).

Wagner et al [38] simulated a form of invasion percolation where the threshold pressures have the form of a self-affine surface. Hence the perimeters of the invaded clusters are the same as the contour lines of the surface. They claim that the perimeter dimension is "consistent" with Isichenko's formula, our Eq. (4.14), but do not quote an error; perhaps their precision was such that the prediction of (4.14) could not have been distinguished numerically from the one we believe to be correct, (4.13).

Ref. [38] also mention measuring a behavior $r^{-\gamma}$ with $\gamma \approx 0.9$ for the correlation between successive filled sites. If this were simply a correlation of two randomly chosen points along a perimeter, it would be identical to our loop correlation functions, $G(r)$ or $G_{s}(r)$ defined in (3.17) or (4.4); in fact, the filling process would appear to depend on correlations of the surface gradient and might have a somewhat different exponent.

\section{Surfaces with a finite correlation length}

To test the percolation analysis of self-affine rough surfaces with a cutoff, as derived in Appendix B and summarized in Sec. IVE, we performed curvature and loop measurements on Gaussian surfaces with a correlation length $\xi$. The correlation length is incorporated in the Fourier method of generating Gaussian surfaces by changing the variance of $\tilde{h}(\mathbf{q})$ in Eq. (5.1) to:

$$
\left\langle|\tilde{h}(\mathbf{q})|^{2}\right\rangle= \begin{cases}|\mathbf{q}|^{-2(1+\alpha)} & \text { for }|\mathbf{q}|>\pi / \xi_{q} \\ \left(\pi / \xi_{q}\right)^{-2(1+\alpha)} & \text { for }|\mathbf{q}| \leq \pi / \xi_{q}\end{cases}
$$

The effects of the cutoff are summarized in Fig. 10, which should be compared to the theoretical prediction of Fig. 2. The curvature and loop data shown in the figure are for system size $L=512$.

The second moment of the curvature displays self-affine scaling with roughness $\alpha=0.4$ up to a length scale set by $\xi_{q}$, and beyond this scale it levels off; see Fig. 10a). We checked that the third moment of the curvature vanishes, as expected since the height fluctuations are still Gaussian, while the fourth moment follows affine scaling up to roughly the same correlation length as the second moment.

The loop measures exhibit distinct crossover behavior, as seen in figures Fig. 10 b) through d). For loops whose radius is smaller than the correlation length, which is here $\xi \approx 20$, we find values of the geometrical exponents consistent with those extracted previously for $\alpha=0.4$ random Gaussian surfaces. For loops whose linear size exceeds the cutoff, scaling consistent with the percolation analysis is found. The actual numerical values extracted by fitting the $20<R<200$ data to a power law are somewhat larger than expected $\left(2 x_{l}=1.46(6), D_{f}=1.7(1)\right.$, and $\tau=2.63(1))$ which we attribute to finite size and/or crossover effects. To check this we also simulated a Gaussian surface with completely uncorrelated heights, i.e., with $\xi=1$ and system size $L=512$, for which we find (by the direct-fit method):

$$
2 x_{l}=1.26(3), D_{f}=1.70(2), \tau=2.565(10)
$$

in good agreement with Eq. (4.16).

\section{SIMULATION: NON-EQUILIBRIUM GROWTH MODEL}

In this section, the linear and nonlinear roughness measures of Sec. III, which in Sec. V were tested on artificial Gaussian random surfaces, are now applied to growth-roughened surfaces produced by a simple random deposition model, the well-known "single-step model". Our results are in support of the view that the single-step model produces self-affine morphologies. 


\section{A. The single-step model}

We implemented the "single-step model" 40 42 in $d=2+1$ dimensions [43]. (More details on this model are found in Sec. III F of Ref. 40], or Sec. II A of Ref. 42].) There is one control parameter $p_{+}$. The allowed configurations are just those of the BCSOS model: each site of a square lattice has an integer-valued height and neighboring heights must differ by \pm 1 . The Monte Carlo rule is that in each time step a deposition event occurs with probability $p_{+}$or an evaporation event (inverse of a deposition event) occurs with probability $1-p_{+}$; once it is decided which type of event occurs, a site is picked at random among those sites at which that event is allowed [44].

We begin by an overview of the theoretical expectations. Up-down symmetry switches $p_{+} \leftrightarrow 1-p_{+}$; thus we need only report data for $0<p_{+} \leq 0.5$. The case $p_{+}=0.5$ is special as the dynamics satisfies detailed balance. This should produce an equilibrium-rough interface, namely the BCSOS model with all configurations weighted equally 45 . This interface, at long wavelengths, is described by the Gaussian model of Sec. V] with $\alpha=0$ (Edwards-Wilkinson behavior).

On the other hand, the growth model for $p_{+} \neq 1 / 2$ is believed to asymptotically belong to the Kardar-Parisi-Zhang (KPZ) universality class 40,42]. It has been proposed that $\alpha=0.4$ exactly for the $2+1$ dimensional KPZ model 46]; however, finite-size effects, small simulations and naive fits systematically underestimate it as $\alpha \approx 0.38$ 47,48. The KPZ behavior should be clearcut when $p_{+}$is close to 1 , but otherwise a crossover from initially Gaussian to asymptotic KPZ behavior is expected, which will be slow (as a function of time or system size) if $p_{+}$is close to $1 / 2$.

It turns out, in our numerical results (below), that $p_{+}=0.5$ indeed shows Gaussian behavior and $p_{+}=0.1$ shows KPZ-like behavior, but $p_{+}=0.3$ consistently resembles $p_{+}=0.5$, at the sizes we could simulate (i.e. up to $L=128$ ). We attribute this to above-mentioned crossover from initial Gaussian behavior.

\section{B. Simulations}

Starting from a flat surface, we ran the simulation (for systems of $128 \times 128$ sites) for 2000 Monte Carlo steps (MCS) per site to equilibrate and then took data for a period of $1200 \mathrm{MCS} /$ site; one such run took 10-15 hours of cpu time on a RISC-6000 workstation. The standard-length runs (for size $L=128$ ) appeared to be insufficiently equilibrated for $p_{+}=0.1$, since they failed to collapse on finite-size-scaling plots with smaller systems. Therefore we performed one run for $L=128, p_{+}=0.1$ with $12000 \mathrm{MCS} /$ site equilibration and $10000 \mathrm{MCS} /$ site for data collection; this is the run reported in our results. In all other cases, we believe the run time was adequate, since much shorter runs showed no gross differences. We performed about four runs for each value $p_{+}=0.1,0.3$, and 0.5 , verifying the symmetry $p_{+} \leftrightarrow 1-p_{+}$. (All measures are the same, apart from a change in sign of $\left\langle C_{b}^{3}\right\rangle$.) Only one of the $\sim 4$ runs was selected to be fitted and plotted here; the data sets presented as e.g. $p_{+}=0.1$ are actually $p_{+}=0.9$ in some cases.

Once every $100 \mathrm{MCS} /$ site (during the data-collecting portion of a run), we performed a measurement step on the surface. The Fourier transform was taken of $h(\mathbf{r})$ using a fast-Fourier-transform routine, but $\left\langle|h(\mathbf{q})|^{2}\right\rangle$ was accumulated only for $\mathbf{q}$ values along the $(1,0),(0,1),(1,1)$, and $(1,-1)$ directions. Also, in each measurement step 100 contour loops were traced out from random initial points, as described in Sec. VC and Appendix C. Statistics were accumulated of the loop's radius $R$ and its number of sites (length) $s$, but not the loop correlation function.

\section{Fourier and curvature results}

The single-step model is the only one simulated in this paper for which we evaluated Fourier spectra, which are plotted in Fig. 11; the log-log plot should have a slope $-2(1+\alpha)$ so $\alpha$ can be extracted from a linear fit (as in Table II). Notice how the spectra are completely isotropic with respect to the lattice directions.

The scale-dependent curvature moments were not evaluated during the runs, but were computed only from the final surface from each run (hence their statistics are much worse than for other measures reported here). We computed $\left\langle C_{b}(\mathbf{x})^{m}\right\rangle$ as defined in Sec. [II A, for $m=2,3,4$, as a function of the offset $b$ in the definition of $C_{b}$ as a discrete Laplacian. The results for $m=2$ and 3 are plotted in Fig. 12. (All figures of the SSM are from the largest system size, $L=128$.) Fig. 12(a) does not show well-defined power laws. The curve for $p_{+}=0.5$ shows a smallish apparent slope, $2 \alpha=0.3(1)$, and a downwards curvature which is plausibly consistent with the expected logarithmic behavior, just like that of the usual height-difference function $D_{2}(b)$ (recall Eq. (3.3). The $\left\langle C_{b}^{2}\right\rangle$ curve for $p_{+}=0.1$ shows a larger slope, $2 \alpha \approx 0.6(1)$, consistent with $\mathrm{KPZ}$ scaling. The $\alpha$ values in Table II], were extracted from fits to $\left\langle C_{b}^{2}\right\rangle$ plots. Slopes from plots of the $\left\langle C_{b}^{4}\right\rangle$ moments (not shown) are consistent with $4 \alpha$ for the $\alpha$ values in the table. 


\section{Loop analysis}

We analyzed the loop ensemble to plot the mean loop size as a function of its radius (Fig. 13) and the cumulative loop-size distribution $P_{>}(s)$ (Fig. 14). (Note that $s$ must have even values; thus it is necessary to divide the nonzero values by 2 in order to properly estimate $P(s)$, which was assumed (in Sec. [II and Sec. [IV] to be a smooth monotonic function.)

As with the Gaussian simulation of Sec. $\mathrm{V}$, the slopes of straight-line fits to log-log plots of these data, give estimates of $D_{f}$ and $\tau-2$; they are tabulated as "direct" in Table [I]. Alternatively, we used data like Fig. 14 from smaller sizes $L=32$ and $L=64$ (as well as $L=128$ ) to produce scaling plots analogous to Fig. 7 (these plots not shown), extracting the "FSS" data in Table II. (We do not report on $2 x_{l}$ since we did not evaluate the loop correlation $G(r)$ in our SSM simulations.)

It is interesting to compare the four different measures of $\alpha$ included in Table II. Those from \langle|$h\left(\left.\mathbf{q}\right|^{2}\right\rangle$ seem to have the smallest statistical errors (and the most sensible values). The closely related $\left\langle C_{b}^{2}\right\rangle$ result is expected to be worse, not only because the statistics are poor in our implementation of the SSM simulation (see above), but also because it uses $h(\mathbf{x})$ values from more widely spaced $\mathbf{x}$ and is therefore more sensitive to the system size.

The next best method seems to be the $D_{f}$ loop analysis from $(\langle s\rangle, R)$ plots; curiously, it appears that the $D_{f}$ fits show smaller run-to-run fluctuations than the \langle|$h\left(\left.\mathbf{q}\right|^{2}\right\rangle$ fits. As also observed in the Gaussian runs (Sec. $\left.\bigvee\right)$, the $P_{>}(s)$ analysis showed more obvious finite-size effects; direct fits to $\tau$ are unreliable and only finite-size scaling plots give reasonable results.

\section{ANALYSIS OF EXPERIMENTAL DATA}

In this section we test our nonlinear measures against experimental scanning tunneling microscopy (STM) data sets. Rough metal surfaces grown under several conditions are believed to develop a morphology with self-affine scaling, but only up to a time-dependent correlation length $\xi(t)$ as discussed in Sec. IV E.

The most detailed analysis was done for the vapor deposited Ag surface on a quartz substrate of Palasantzas and Krim 49]. We obtained a $400 \times 400$ height array corresponding to an STM image of a $702 \mathrm{~nm}$ thick Ag surface and performed curvature and loop measurements. Note that all the results quoted below are from a single height profile. All in-plane lengths will be measured in units of the grid of this data, which is $1.625 \mathrm{~nm}$.

We also report briefly (Subsec. VIIC) a less thorough analysis of an STM data set from a different, but still self-affine, growth regime showing KPZ scaling.

\section{A. Quadratic measures and curvature moments}

Palasantzas and Krim 49] originally evaluated the roughness exponent

$$
\alpha=0.82(5)
$$

from a fit to the standard (quadratic) height correlation function $D_{2}(\mathbf{r})$ defined in Eq. (2.2).

That correlation is similar to our second curvature moment $\left\langle C_{b}^{2}\right\rangle$. This quantity shows a power law dependence on the scale $b$, up to a correlation length which was estimated to be $\xi=25(5)$; see Fig. 15(a). A linear least squares fit of the data with $b<\xi$ gave $2 \alpha=1.7(1)$, agreeing (as expected) with (7.1).

The third moment $\left\langle C_{b}(\mathbf{x})^{3}\right\rangle$, Fig. 15(b), shows distinct non-Gaussian behavior, as expected for non-equilibrium growth. It reaches a maximum at length scale $b \approx 23$ which correlates well with $\xi$. This indicates a morphology consisting of grains of typical size $\xi$ that are rounded at the top. Such a morphology is clearly seen in three-dimensional renderings of the STM data in Ref. 49, or the gray-scale image in Ref. [50].

\section{Kleban's nonlinear measure}

Recently, Kleban et al defined a non-quadratic roughness measure rather different from any of those mentioned in Sec. III. First, for every scale $b$ they constructed a smoothed version $H_{b}(\mathbf{x})$ of the height function, as the average of $h\left(\mathbf{x}^{\prime}\right)$ for $\mathbf{x}^{\prime}$ in a $b \times b$ square centered at $\mathbf{x}$ (alternatively by convolving with a Gaussian weight function of width b.) Then they calculated the histogram $P\left(H_{b}\right)$ of $H_{b}(\mathbf{x})$ values for $\mathbf{x}$ ranging over the entire sample, and the skew moment of this distribution. (Of course, $P\left(H_{b}\right)$ is defined for a rough surface only when a finite-size or finite-time cutoff is present.) 
When applied to the STM data of Palasantzas and Krim [49], the distribution of $H_{b}(\mathbf{x})$ appears Gaussian when $b=0$, i.e. for the raw data. That is rather mysterious, since the surface certainly lacks up/down symmetry: it consists of deep, narrow crevasses and rounded hills. Each crevasse should contribute to a long tail on only the $h<\bar{h}$ side of the distribution, while each hill contributes a peak and a sudden drop to zero on the $h>\bar{h}$ side. However, the fluctuation in height from hilltop to hilltop smears out that sharp feature, making a spuriously symmetric distribution. As pointed out in Ref. [50], their smoothing of $h(\mathbf{x})$ eliminates the deep crevasses so the smoothed surface $H_{b}(\mathbf{x})$ does have a skewed height distribution (with skewness dependent on the observation scale $b$ ). This is consistent with our own conclusion that the height fluctuations are non-Gaussian.

\section{B. Contour-loop analysis}

We perform loop measurements and check whether the different scaling relations derived in section IV are satisfied. The moments of the scale dependent curvature are used as an independent measurement of the roughness and to assess the Gaussianness of the height fluctuations.

Using the loop algorithm (appendix G), we measure the loop radii and corresponding loop lengths for 1000 contour loops constructed through randomly chosen points on the surface; however, we did not compute the loop correlation function. These loop measures support the scenario that the surface is self-affine up to a correlation length $\xi \approx 25$.

The average loop length is plotted against the loop radius in Fig. 16. We see a decade of power law scaling of the length with the radius, and from a linear least squares fit of the data to a line, for $5<R<50$, we find

$$
D_{f}=1.06(2)
$$

for the fractal dimension of contour loops. Using the formula for $D_{f}$, Eq. (4.13), we calculate $\alpha=0.88(4)$ in good agreement with the reported value, Eq. (7.1). In Fig. 16 the dashed line corresponds to the percolation value $D_{h}=1.75$; we see that loops at scales much larger then $\xi$ show scaling consistent with this value.

Finally, the number of loops whose length exceeds $s, P_{>}(s)$, is plotted in Fig. 17. The data roughly shows two scaling regimes with different exponents, before it is cut-off by the system size. The knee occurs at loop lengths $s \approx 70$ which, from Fig. 16, corresponds to a loop radius of 20 or so; this again is comparable to the length scale $\xi \approx 25$ found from the curvature data. From loops whose length is in the interval $(10,30)$ we extract the exponent

$$
\tau-2=0.069(5)
$$

while larger loops exhibit scaling consistent with the percolation value (indicated by the dashed line). Using Eq. (4.13) we find $\alpha=0.85(1)$ again in good agreement with the self-affine exponent reported by Palasantzas and Krim.

To summarize, the two loop measures we evaluated, as well as quadratic scale-dependent curvature, all indicate self-affine scaling with a roughness exponent $\alpha \approx 0.85$, up to a length scale $\xi \approx 25$. Beyond this scale the height fluctuations appear to be uncorrelated.

\section{Other data sets}

The large value of $\alpha$ found for the silver-on-quartz STM data of Palasantzas and Krim is indicative of MBE-type growth which has surface diffusion as a dominant relaxation process. This motivates the study of other data sets which might correspond to different universality classes of growth. For example, the KPZ equation describes growth dominated by desorption and/or vacancy formation, both of which are relaxation processes that do not conserve particle number [10,11].

Loop measurements were carried out previously on gold electro-deposits by Gomez-Rodriguez et al [51]. These authors suggested the fractal dimension of contour loops as a useful measure for characterizing the surface morphology. What was lacking in their analysis was an equation relating $D_{f}$ to the roughness exponent $\alpha$. From STM images of deposits grown in the fast and slow regime they determined the fractal dimension to be $D_{f} \approx 1.5$ and $D_{f} \approx 1.3$, respectively. Now using Eq. (4.13) we calculate the roughness in these two regimes to be: $\alpha \approx 0$ and $\alpha \approx 0.4$. The first is expected for Edwards-Wilkinson type of growth $(\alpha=0)$, while the second is in good agreement with the Kardar-Parisi-Zhang value $\alpha=0.38$ (from most fits) or 0.40 (possibly exact) 47, 48 ].

Csahok et al. [52] studied the surface morphology of Ni films vapor-deposited on a quartz substrate. (They were interested mainly in the effects of subsequent ion sputtering on the film.) We obtained an STM image of the as-grown Ni surface (before any sputtering) in the form of a $256 \times 256$ height array, and computed some of the contour-loop measures for it from a collection of 10000 loops. The results are consistent with a (KPZ-like) self-affine morphology 
with a roughness of $\alpha=0.4$. Namely, the loop data shows a limited range of scaling for loop radii $10<R<30$. Direct fits to a straight line of the log-log plots of $\langle s\rangle(R)$ and $P_{>}(s)$ in the scaling regime, yield $D_{f}=1.31(4)$ and $\tau-2=0.22(2)$. After inverting the formulas for $D_{f}(\alpha)$ and $\tau(\alpha)$ in Eq. (4.13) we obtain the estimates $\alpha=0.38(8)$ and $\alpha=0.44(4)$ respectively.

\section{DISCUSSION}

Here we summarize our main results, compare and critique previously introduced measures of surface roughness, and describe some open problems and interesting new directions.

\section{A. Summary of results}

We introduced (in Sec. III) new measures for characterizing the spatial correlations of rough interfaces. Their common property is that they are not linearly related to the structure function of the height. First, we introduced the scale dependent curvature. Its third moment is an indicator of the skewness of the height distribution, and thus is a good criterion for whether or not a surface's height fluctuations are Gaussian. Our chief focus, though, was on the ensemble of contour loops as a novel means of characterizing surfaces. For a rough self-affine surface, the loop ensemble is critical and we introduced three kinds of geometrical exponent associated with it: $x_{l}$ for the loop correlation function (probability that two points are on the same contour loop), the fractal dimension of a contour loop, $D_{f}$, and $\tau$ associated with the length distribution of loops. In particular, we conjectured a super-universal value $2 x_{l}=1$ (see Sec. IVQ) which has been confirmed so far numerically (e.g. in Table I), but not analytically. The loop exponents satisfy scaling relations (derived in Sec. IV), and granting the conjecture, their values are completely determined by the affine (roughness) exponent $\alpha$.

Next, we showed how numerical values of the geometrical exponents can be extracted in practice from height data obtained from simulations or experiments. We first did this in Sec. \ for artificial Gaussian surfaces (known analytically to be self-affine) and in Sec. VI for configurations from simulations of the single-step (growth) model (believed to be self-affine); this served as a check to confirm the validity of our scaling relations. Then in Sec. VII we processed an experimental data set - an STM image of a growth roughened silver film 449 - in the same fashion. The results here also confirmed the scaling relations which in this case adds to the evidence of the self-affine nature of the height fluctuations. The third moment of the scale-dependent curvature confirmed that the height fluctuations are non-Gaussian, while the contour-loop fractal dimension and size distribution indicated self-affine scaling with $\alpha \approx 0.85$.

Experimental data often exhibit self-affine scaling up to a correlation length $\xi(t)$. We argued (in Sec. IVE) that the loop exponents, for loops whose linear size exceeds the correlation length, are determined by exactly known percolation exponents. The crossover between the self-affine and percolative regime was visible (with a consistent $\xi$ value) in every kind of measure on the experimental data in Sec. VII - the same was true for Gaussian random surfaces with an artificial length scale cutoff (Sec. VD). The numerical values of the percolative exponents were confirmed from simulations of Gaussian surfaces with a white-noise spatial power spectrum.

Our results (see Sec. $\mathrm{V}$ ) show that it is quite difficult to get correct results from loop measurements when $\alpha$ is near to 1 . The reason, we believe, is that the crossover to asymptotic behavior occurs at very large loops; the inferred $\alpha$ is thus smaller than the real one. It has been observed [53] that even the height-height correlation function tends to yield a too small value of $\alpha$ as compared to the Fourier power spectrum, even though the two measures have, in principle, the same information.

\section{B. Comparisons of roughness measures}

Roughness has often been analyzed based on a single number, the overall variance of the height over the entire system. However, spatial correlations in height fluctuations are central to the development of self-affine or other interesting morphologies. Therefore, every form of roughness measure we discuss takes the form of a spatial spectrum, i.e., one measures an entire function whose argument has dimensions of length (called $s, R, b$, or $1 / q)$. The variation of the roughness measure with its argument is related to the varying amount of interface fluctuations on the corresponding length scales.

Some previous measures of the self-affine exponents were reviewed in Ref. [18]. They systematically compared the different measures using artificially constructed realizations of $h(x)$ (only in $1+1$ dimensions), and concluded that 
the single best measure of $\alpha$ is the Fourier power spectrum, our Eq. (2.4). (Oddly enough, Ref. [18] did not include the height correlation function, our Eq. (2.2), in their selection of measures to compare.)

Another approach is to measure $h(\mathbf{x})$ along a single line in the $\mathbf{x}$ plane, corresponding to a line scan by the STM [54. This section through the surface may then be analyzed as if it were a $1+1$-dimensional profile. Ref. [54] evaluated the variance over an interval of length $L_{0}$, which should scale as $L_{0}^{2 \alpha}$, and applied this experimentally to the heteroepitaxy of $\mathrm{CuCl}$ on the (111) surface of $\mathrm{CaF}_{2}$.

The most useful roughness measures have been discussed and critiqued in the sections related to them; they fall into three categories and are summarized here in Table [II].

\section{Quadratic roughness measures}

The most familiar measures are quadratic of which the first three were summarized in Sec. IIB 1. Besides three well-known quadratic measures, we include a fourth which has not been previously applied: the variance of scaledependent curvature, $\left\langle C_{b}(\mathbf{x})^{2}\right\rangle$, which we introduced in Eq. (3.3) (Of course, the ensemble expectation cannot depend on $\mathbf{x}$.) Its behavior is very similar to that of the height-difference correlation $D_{2}(\mathbf{r})$, so $\left\langle C_{b}^{2}\right\rangle$ is of interest mainly for comparison with the higher moments of $C_{b}(\mathbf{x})$. In practical applications the Fourier spectrum is probably the best of these.

A key fact about the quadratic measures is that, given the complete function for any one of them, one can compute the complete function for any other one as a linear transform (convolution with some kernel) This property is not true for higher moments. Notice also that the quadratic measures are invariant under $h(\mathbf{x}) \rightarrow-h(\mathbf{x})$ and so cannot possibly characterize the breaking of up/down symmetry in the growth process. Nor can they identify deviations from Gaussianness, since one can produce a Gaussian ensemble (as in Sec. VA) with any given Fourier spectrum.

\section{Non-quadratic roughness measures}

Essentially all of these have been developed by analogy with quadratic measures, simply replacing the second power by a higher power. Our curvature-moment function seems to be the first generalization of the height-difference function that captures the up/down asymmetry.

A simple generalization of the $b$-box variance is the $b$-box $q$-th moment, $\left\langle\left(h(\mathbf{r})-\bar{h}_{b}\right)^{q}\right\rangle_{b}$. The $q=3$ moment characterizes the up/down asymmetry; when scaled by $\left\langle\left(h(\mathbf{r})-\bar{h}_{b}\right)^{2}\right\rangle_{b}^{3 / 2}$ it defines a scale-dependent, dimensionless skewness that measures the deviation from Gaussianness [55]. This appears to be a simple and attractive measure, but we know of no applications to date; our curvature moment $\left\langle C_{b}^{3}\right\rangle$ is similar in spirit, but probably not linearly related.

We evaluated the quartic curvature moment $\left\langle C_{b}^{4}\right\rangle$, but this data was less useful than our other measures: it does not reveal the non-Gaussian nature as strikingly as $\left\langle C_{b}^{3}\right\rangle$ does. The dimensionless ratio $\left\langle C_{b}^{4}\right\rangle /\left\langle C_{b}^{2}\right\rangle^{2}$ is 3 in the Gaussian case, but may not differ very much in a non-Gaussian ensemble. Furthermore, the roughness exponent was fitted less precisely from $\left\langle C_{b}^{4}\right\rangle$ than from any other measure, probably due to the sensitivity of higher moments to rare events.

The analysis in Ref. [50] summarized in Sec. VII A 1, appears to be the first application of a scale-dependent roughness measure to characterize the up/down asymmetry. However, we believe a local roughness measure such as the $b$-box skewness or (better) our mean cubed curvature gives a more meaningful characterization. In a sense, the smoothed-height skewness is the opposite of the local measures since it includes the fluctuations from all length scales larger than $b$, while the local measures include the fluctuations from scales comparable to or smaller than $b$; only the latter would be expected to scale as $b^{\alpha}$.

\section{Loop measures}

The other non-quadratic roughness measures, of course, are the loop measures defined in Sec. III. The length and connectedness of a loop, manifestly, depend on the heights $h(\mathbf{r})$ in a highly nonlinear fashion, and one might expect the loop exponents to be independent of the roughness exponent $\alpha$; then the loop properties might have distinguished between different universality classes of growth which happen to have similar $\alpha$ values. From this viewpoint, it is disappointing that we in fact find the loop exponents are functions of $\alpha$ (Sec. IV). Thus for self-affine interfaces the loop measurements serve only as a check on other ways (quadratic and non-quadratic) of measuring $\alpha$. Furthermore, when the heights at large separations are uncorrelated, implying the loops are percolation hulls (see Sec. IVE), the loop plots show a weaker change of slope at this crossover than the Fourier spectrum does. 
It seems worthwhile nevertheless to compute loop measures. In a sense they depend on higher order correlation functions of the heights: then the agreement between the $\alpha$ values extracted from loops and from other measures is an additional, stringent test of self-affineness. Also we observe empirically that loop measures, and in particular the average loop length as a function of loop radius, are very self averaging and measurement of $\alpha$ from them produces smaller errors than either the real-space or Fourier-space methods. Finally, although the loop exponents are the same for different universality classes with the same $\alpha$, we do expect universal coefficients to be different.

For computer generated height data the loop-size-distribution is, perhaps, the single most valuable plot, because two different exponents can be obtained from scaling plots such as Fig. 77. This is not the case for experimental data where the system size is typically not a tunable parameter.

The loop-correlation function $G(\mathbf{r})$ is most tedious to compute, and since its exponent $2 x_{l}$ is superuniversal it does not yield an estimate of $\alpha$. Nevertheless $G(\mathbf{r})$ is a useful check on the self-affineness, since the superuniversal behavior fails in other cases (e.g. beyond the correlation length, see Eq. (4.16)).

\section{Future directions}

New experimental techniques which provide complete real-space images of the fluctuating quantity of interest (rather than system-wide averages, or local measures probing the system at only a few points), are being developed in every physical science. Consequently, measures which usefully exploit this wealth of information will gain in importance. In turn, the ability to measure new (and nonlinear) correlations may inspire new theories that can predict the correlation behavior.

\section{Turbulence}

Fluid dynamics is a good example of the interplay just mentioned between theory and experiment: formerly two (sometimes more) point correlations were measured by hot-wire probes, and the same correlations were the objects of the Green's function method. As full images become available of the velocity field, many new measures are attempted in order to capture more of the available information.

Indeed, the measures introduced here might be adapted to the geometrical description of turbulence. The advection of passive tracers by turbulent flows seems a to be an especially promising problem. There is already considerable interest in characterizing the equal-time correlations through fractal measures of the contours of (say) constant tracer concentration [56,50,57,6. (To maintain the analog of a surface's symmetry under global shifts of the height, one should study the logarithm of the concentration and use contours spaced equally on the logarithmic scale.)

Measurements of the fractal dimension of iso-concentration lines of a passive tracer advected by a magnetically driven, turbulent, two-dimensional flow were reported by Cardosa et al. [6]. They find $D_{f}=1.35(5)$, which, assuming the concentration field is self-affine, yields a roughness exponent $\alpha=3-2 D_{f}=0.3(1)$. Indeed, $\alpha=0.30(3)$ was measured by the authors, by applying the $q=1$ multiaffine correlation measure (entry 6 . in Table III). We therefore infer that their measurements are consistent with a self-affine morphology for the concentration field. Details of the complete loop and curvature analysis of this data set will be reported in a separate paper [58].

\section{Other dimensions?}

In this connection, it is interesting to consider the generalizations of $h(\mathbf{x})$ to spatial dimensions of $\mathbf{x}$ other than 2. In the $1+1$-dimensional case, there are no loops; the probability of first return to a fixed height value [18] seems to be the closest analog to our loop size distribution of Subsec. III (if presented as a distribution of $R$ instead of $s$ ) and also to our loop correlation function Eq. (3.17).

With each higher dimensionality there is greater richness in distinct geometrical measures that can be defined for iso-surfaces. For a hypersurface in 3+1 dimensions - like the concentration function in three-dimensional passive tracer advection - the level set may be multiply connected and even knotted. Nevertheless the size distribution exponent $\tau$, the fractal dimension $D_{f}$, and $x_{l}$ of the connectedness correlations, can be generalized directly. But we see much less reason to expect a super-universal connectedness correlation exponent in dimensions higher than $d=2+1$. 


\section{Multifractality and scaling relations}

Several mysteries remain about the scaling relations derived in Sec. IV. Above all, there is not yet any rigorous or analytic basis for our fundamental conjecture (Eq. (4.12)) of a super-universal loop correlation that scales as $1 / r$ for all rough self-affine surfaces - unaffected even by quenched disorder that further roughens the interface [34]. A second open question is to check numerically the correlation exponent $a$ in Eq. (4.4) for an individual loop of fixed size; we did not evaluate it in any of our numerical studies, but it should not be the same as the exponent $2 x_{l}$ for the ensemble average over loops of all sizes. Finally, it is intriguing to ask what happens in a "multiaffine" system [22,23. Here different moments of the height variables have different scaling exponents; which of these (if any) is the one entering our formulas (4.9) and (4.10) for the loop exponents?

\section{ACKNOWLEDGMENTS}

We would like to acknowledge helpful discussions with M. Aizenman, M. Avellaneda, P. Constantin, D. Fisher, G. Huber, M. Isichenko, T. Spencer, C. Zeng, and particularly to thank J. Krim, M. Plischke and Z. Csahok for supplying STM data, and Michael Plischke for providing his code to simulate the single-step model. We are grateful to M. B. Isichenko for bringing Ref. [39] to our attention, and to J. Gollub for alerting us to Ref. [6]. C.L.H. was supported by NSF Grant No. DMR-9612304, D.G.S. by NSF Grant No. DMR-9632275 through the Cornell Materials Science Center, and J.K. by NSF Grants No. DMS 93-04580, DMS 97-29992, and (during a portion of this work at Brown University) DMR-9357613.

\section{APPENDIX A: ANALYTIC DERIVATION OF $X_{L}(\alpha=0)$ FOR AN EXACT SOLUBLE MODEL}

Our purpose here is to support the conjecture (4.12) in Sec. IV , by showing that $x_{l}=1 / 2$ in the case of a lattice model which can be mapped to an equilibrium-rough surface. At long wavelengths height fluctuations are described by the well-known free energy

$$
F=(\text { constant }) \int d^{2} \mathbf{x}|\nabla h(\mathbf{x})|^{2}
$$

which by equipartition implies Eq. (2.7) with $\alpha=0$, so indeed the surface is self-affine. This appendix only summarizes arguments made previously in Refs. [21], [59], and [60].

Consider a statistical model with microscopic heights $z_{\mathbf{j}}$ defined on a triangular lattice $\{\mathbf{j}\}$, such that $z_{\mathbf{j}}$ changes by 0 or \pm 1 between nearest neighboring sites. The partition function of the model is

$$
Z=\sum_{\{z\}} \prod_{<\mathbf{j}, \mathbf{k}>} w\left(z_{\mathbf{j}}-z_{\mathbf{k}}\right)
$$

where $w(0)=1$ and $w( \pm 1)=K$; the sum goes over all microscopic height configurations unrelated by a global height shift.

A contour-loop configuration $\gamma^{\prime}$ is specified by drawing closed (periodic boundary conditions ensure that all contour lines are closed), oriented, non-intersecting loops along the bonds of the dual honeycomb lattice, which seperate sites that differ in height by \pm 1 (the sign determines the loops orientation). In terms of the loops, the partition function is

$$
Z=\sum_{\gamma^{\prime}} K^{N_{b}}
$$

where $K$ is the fugacity of an occupied bond (i.e. one covered by a loop), and $N_{b}$ is the number of occupied bonds in $\gamma^{\prime}$.

This model is equivalent to the $O(2)$ loop model introduced by Nienhuis [33]. This is seen by rewriting the partition function in terms of non-oriented loop configurations $\gamma$,

$$
Z=\sum_{\gamma} K^{N_{b}} 2^{N_{l}}
$$

where $N_{l}$ is the number of loops in $\gamma$ (which is the same as the number of loops in $\gamma^{\prime}$ ), and the 2 appears as a result of summing over the two possible orientations for each loop in $\gamma^{\prime}$. 
By mapping the $O(2)$ loop model to the 4-state ferromagnetic Potts model on the triangular lattice, Nienhuis showed that for $K_{c}=\sqrt{2}$ the loop model is critical. Using the Coulomb-gas picture of correlations [33] this implies that $\left\{z_{\mathbf{j}}\right\}$ are rough; since the "background charge" is zero [33], it is plausible that (at $\left.K_{c}\right)$ the height model is equilibrium-rough, i.e. the field $h(\mathbf{x})$ obtained by coarse-graining $z_{\mathbf{j}}$ satisfies (A1). Furthermore the contour-loop correlation function can be identified in the $O(2)$ model with the so-called energy-energy correlation function which at $K=K_{c}$ decays as a power law with the known exponent

$$
x_{l}=1 / 2,
$$

as was to be shown.

Since we view the $O(2)$ loop model simply as one of many possible lattice disretization of a logarithmically rough $(\alpha=0)$ self-affine surface, then the exponent $x_{l}=1 / 2$ should necessarily appear in other lattice models that map to rough surfaces. Indeed the same value of this exponent follows also from the exact solution of the $O(2)$ loop model on the square lattice [61], and the $n=2$ fully packed loop model on the honeycomb lattice [62].

\section{APPENDIX B: PERCOLATION SCALING OF CONTOURS FOR UNCORRELATED HEIGHTS}

This appendix derives the scaling behavior of the loop ensemble when the random heights $h(\mathbf{x})$ have a finite variance and (beyond a correlation length $\xi(t)$ ) are uncorrelated; this describes early stages of growth, as in Sec. IVE.

To model the contour loops at length scales greater than $\xi(t)$, first coarse-grain the system into boxes of side $\xi(t)$. The average height $h$ in each box is an independent random variable parametrized by $p\left(h^{\prime}\right)$, the probability that $h<h^{\prime}$.

Defining all the boxes with $h<h^{\prime}$ as "filled" simply reproduces the (uncorrelated) percolation clusters for occupancy $p\left(h^{\prime}\right)$. Then every contour of constant $h^{\prime}$ is simply the perimeter of such a cluster. This mapping is well-known from Ref. [63] and is widely applied in the theory of the quantum Hall effect [35, 63].

The percolation clusters - as well as their perimeters - are self-similar only at $p(h)=p_{c}$, the percolation threshold; we will first discuss their (known) loop exponents. The behavior when $p \neq p_{c}$ can easily be derived from well-known percolation scaling relations. The final step will be to integrate these results over $p$, since the loop ensemble we simulate actually corresponds to the union of perimeter ensembles for all $p$.

\section{Contour loops and critical percolation}

Fixing $p=p_{c}$ for a moment, the perimeter loop ensemble may be characterized by exponents $D_{h}$ and $\tau_{h}$, with definitions analogous to (3.9) and (3.13) for $D_{f}$ and $\tau$. (The subscript " $h$ " stands for "hull" as the perimeter is often called.) The fractal dimension

$$
D_{h}=7 / 4
$$

is known exactly [36].

The perimeter loops for percolation at $p_{c}$ also satisfy a hyperscaling relation analogous to (4.3), with $\alpha$ replaced by zero. That is, the largest cluster (or perimeter) diameter inside a box of side $l$ is least $\sim l$. From this follows a relation for $\tau_{h}$

$$
\tau_{h}=1+2 / D_{h}=15 / 7 .
$$

When $p \neq p_{c}$, the cluster (and perimeter) ensemble scaling is cut off at the percolation correlation length $\xi_{p}(p)$, which diverges near $p_{c}$ as

$$
\xi_{p}(p) \sim\left|p-p_{c}\right|^{-\nu_{p}},
$$

where $\nu_{p}$ is the usual percolation correlation exponent, and $\nu_{p}=4 / 3$ is known exactly [64, 36]. In this case, the loop length distribution is

$$
P_{h}(s ; p)=s^{-\left(\tau_{h}-1\right)} f_{h}\left(s / \xi_{p}(p)^{D_{h}}\right)
$$

where $f_{h}()$ is a scaling function, which falls off exponentially fast for loops of radius greater than $\xi_{p}(p)$. 


\section{Union of all percolation contours}

In the percolation regime, evidently, the statistical properties of the contours of a particular level set depend on the chosen level $h$. (This was impossible in the self-affine regime, since in that case the fluctuations of $h$ were unbounded.) But we have previously studied the union of all contours with different $h$, corresponding to all values of $p(h)$ from 0 to 1 . That is, indeed, the ensemble sampled by our computer codes (see Sec. V q). We will now derive the exponents $\tau_{p}$ and $x_{l, p}$ of this ensemble, defined analogously to $\tau$ and $x_{l}$ in eqs. (3.13) and (3.17).

Most of the loops at a large length scale $R$ come from levels sets at height $h$ with $\xi(p(h))>R$, rather than from the exponential tails of the distribution (B4) for the other $h$ values. Thus these obey the percolation scaling and all have fractal dimension $D_{f, p} \equiv D_{h}$. This behavior is illustrated in Fig. 2(c).

Now, in the percolation regime, $P(s)$ as defined in Sec. III , is just proportional to the integral of $P_{h}(s ; p(h))$ over h. A weighting factor $|d p / d h|$ should be included as the contours are equally spaced, and $p(h)$ is normalized to unity. Since the large contours come from $p \approx p_{c}$, only that part of the distribution matters. Inserting (B3) into (B4), one obtains

$$
P(s) \sim \int d p s^{-\left(\tau_{h}-1\right)} f_{h}\left((\text { const }) s\left|p-p_{c}\right|^{D_{h} \nu_{p}}\right)
$$

hence $P(s) \sim s^{-\left(\tau_{p}-1\right)}$ with

$$
\tau_{p}=\tau_{h}+\left(D_{h} \nu_{p}\right)^{-1}=18 / 7
$$

Finally, given $(\mathrm{B} 6)$ the simplest route to the loop (connectedness) correlation exponent is to use the exponent relation 4.8$)$; this gives

$$
2 x_{l, p}=4-2 D_{h}+\nu_{p}^{-1}=5 / 4 .
$$

Eq. (B7) could alternately be reached by first noting that the corresponding exponent is $1 / 2$ for the percolation hull ensemble at $p_{c}$, and then averaging the loop connectedness correlation function analogous to (B5).

\section{APPENDIX C: LOOP FINDING ALGORITHM}

Given a square lattice $\mathcal{L}$ on which the heights $h$ are defined and a point $\mathbf{x}_{0}$ on the dual lattice $\mathcal{L}^{*}$, the task of the loop finding algorithm is to construct a contour loop of the surface which passes through the point $\mathbf{x}_{0}$.

The contour is a walk along the bonds of $\mathcal{L}^{*}$ that cuts those bonds of $\mathcal{L}$ that have vertices with heights lying above and below the contour height, Fig. 18. To implement this idea we first define the level height $h_{\text {lev }}$ which is the average of the four heights around the plaquette centered at $\mathbf{x}_{0}$. Second, we assign to all the sites of $\mathcal{L}+$ or - signs according to whether they are above or below the chosen level $h_{\mathrm{lev}}$. Now, starting from $\mathbf{x}_{0}$ we form the contour loop by drawing links on the dual lattice which cross the bonds of $\mathcal{L}$ connecting + and - sites. This is repeated until the walk returns to the starting point $\mathbf{x}_{0}$; the finite extent of the lattice $\mathcal{L}$ is dealt with by implementing periodic boundary conditions.

Special care must be taken whenever a "saddle-point" plaquette is reached, that is one where the sites of the lattice are assigned +-+- signs cyclically around the plaquette. In this case four links meet at the point in the center and we must resolve the connectivity there by an additional rule, so as to convert this pattern into two 90-degree turns that are not quite touching. One natural condition on the rule is that it should be reversible, that is one should find the same loop whether one starts traversing it clockwise or counterclockwise. A second condition is that it ought to be invariant under reflecting all heights by $h(\mathbf{x}) \rightarrow-h(\mathbf{x})$. A physically sensible rule which satisfies both conditions makes use of the average height $h_{\text {plaq }}$ of the four heights around the saddle-point plaquette. If $h_{\text {plaq }}<h_{\text {lev }}$, we view the center of the plaquette as being lower than the level of the contour loop and the connectivity is resolved by having the + sites inside the 90-degree turns. In the opposite case, $h_{\mathrm{plaq}}>h_{\mathrm{lev}}$, the + sites lie outside the 90 -degree turns; see Fig. 18. (The agreement of loop data from the single-step model with parameters $p_{+}$and with $1-p_{+}$, as explained in Sec. VI was a valuable check of the up-down symmetry of our loop-finding algorithm.)

Once a contour loop through $\mathbf{x}_{0}$ has been constructed its length and radius are recorded, assuming that the loop is topologically trivial. (Due to periodic boundary conditions loops with non-zero winding numbers are possible and these we discard.) The contour loop length $s$ is equal to the number of steps made during the loop construction, while the radius $R$ is the size of the largest square which covers the loop, i.e., the maximum displacement in the $x$ or $y$ direction. Every topologically trivial loop also contributes to the correlation function $G(r)$; for every point on the loop that is a distance $r \in[i, i+1)$ ( $i$ is an integer) away from the starting point $\mathbf{x}_{0}$, the array element $g(i)$ is 
increased by one. We define $G(r)$ for our simulations as $g(r) / 2 \pi r$, which asymptotically is normalized the same as $G(r)$ defined in Sec. III .

So far we have assumed that the height variables are real and the condition $h_{\text {plaq }}=h_{\text {lev }}$ is almost never fulfilled. This is not the case for interfaces which arise from discrete growth simulations like the one presented in section VI where the height variable takes on integer values. In this case the resolution of the connectivity should be completely random but we must ensure that we use the same choice if the loop returns to the same plaquette. The simplest way to do this, which is what we have implemented, is to take the original integer heights and "dither" them - add small amounts of random, uncorrelated gaussian noise to all $h(\mathbf{x})$. This will also solve the problem of choosing $h_{\text {lev }}$; it will be non-generic for any two heights to precisely coincide, although this will happen occasionally as the price of roundoff error. When this does happen we start over by choosing a new initial site $\mathbf{x}_{0}$.

[1] Present address: Department of Physics MS057, Brandeis University, Waltham, MA 02254.

[2] Present address: 3476 Avenue Laval, Montréal, Québec, H2X 3CB Canada.

[3] E. Bouchaud, G. Lapasset, and J. Planès, Europhys. Lett. 13, 73 (1990); Phys. Rev. B 48, 2917 (1993).

[4] W.B. Wright, R. Budakian, D.J. Pine, and S.J. Putterman, Science 278, 1609 (1997).

[5] R. Ramshankar and J.P. Gollub, Phys. Fluids A 3, 1344 (1991).

[6] O. Cardosa, B. Gluckmann, A. O. Parcollet, and P. Tabeling, Phys. Fluids 8, 209 (1996).

[7] S. Lovejoy, Science 26, 185 (1982).

[8] J. D. Pelletier, Phys. Rev. Lett. 78, 2672 (1997).

[9] J. P. Grotzinger and D. H. Rothman, Nature 383, 423 (1996).

[10] G. Palasantzas and J. Krim, Int. J. Mod. Phys. B9, 599 (1995).

[11] J. Krug, Adv. Phys. 46, 139 (1997).

[12] Height configurations have also been derived (for buried interfaces) from dark-field transmission-electron-microscope images: see X. Chen and J. M. Gibson, Phys. Rev. Lett. 81, 4919 (1998).

[13] Our treatment is limited to single-valued height field; thus coarse graining of $h(\mathbf{x})$ is mandatory when overhangs are present at atomic length scales, and we do not consider the case that overhangs persist to large scales.

[14] A.-L. Barabási and H. E. Stanley, Fractal Concepts in Surface Growth (Cambridge University Press, Cambridge, 1995); see also T. Halpin-Healy and Y.-C. Zhang, Phys. Rep. 254, 215 (1995).

[15] S. J. Chey, J. E. van Nostrand, and D. G. Cahill, Phys. Rev. Lett. 76, 3995 (1996).

[16] E. A. Eklund, R. Bruinsma, J. Rudnick, and R. S. Williams, Phys. Rev. Lett. 67, 1759 (1991).

[17] L. Vásquez, J. M. Vara, and E. Ziegler, J. Appl. Phys. 75, 248 (1994); T. Holten, T. Jøssang, P. Meakin, and J. Feder, Phys. Rev. E 50, 754 (1994).

[18] J. Schmittbuhl, J.-P. Vilotte, and S. Roux, Phys. Rev. E 51, 131 (1995).

[19] B. B. Mandelbrot, The Fractal Geometry of Nature (Freeman, New York, 1982).

[20] M.S. Spector, E. Naranjo, S. Chiruvolu, and J.A. Zasadzinski, Phys. Rev. Lett. 73, 2867 (1994).

[21] J. Kondev and C. L. Henley, Phys. Rev. Lett. 74, 4580 (1995).

[22] J. Krug, Phys. Rev. Lett. 72, 2907 (1994).

[23] S. Das Sarma and P. Punyindu, Phys. Rev. E 55, 5361 (1997); C. Dasgupta, J. M. Kim, M. Dutta, and S. Das Sarma, Phys. Rev. E 55, 2235 (1997).

[24] G. Stolovitzky and K. R. Sreenivasan, Rev. Mod. Phys. 66, 229 (1994).

[25] S. Das Sarma, C. J. Lanczycki, R. Kotlyar, and S. V. Ghaisas, Phys. Rev. E 53, 359 (1996).

[26] D. Stauffer and A. Aharony, Introduction to Percolation Theory (2nd ed.), (Taylor \& Francis, London, 1992).

[27] For lattice models that map to logarithmically rough Gaussian surfaces, the probability that two loops with radii larger than $R$ have a distance of closest approach smaller than $r$ vanishes as a power of $r / R$ (Ref. [36]).

[28] G. Huber, M. H. Jensen, and K. Sneppen, Fractals 3, 525 (1995).

[29] M.B. Isichenko and J.L. Kalda, J. Nonlinear Sci. 1, 255 (1991).

[30] M. Aizenman, Nucl. Phys. B485, 551 (1997).

[31] The equality in Eq. (4.5) depends on $s$ and $G$ being defined with compatible normalizations, which is the case for lattice height models.

[32] Z. Olami and R. Zeitak, Phys. Rev. Lett. 76, 247 (1996).

[33] B. Nienhuis, in Phase Transitions and Critical Phenomena, edited by C. Domb and J.L. Lebowitz (Academic, London, 1987), Vol. 11.

[34] C. Zeng, J. Kondev, D. McNamara, and A. A. Middleton, Phys. Rev. Lett. 80, 109 (1998).

[35] M. B. Isichenko, Rev. Mod. Phys. 64, 961 (1992).

[36] H. Saleur and B. Duplantier, Phys. Rev. Lett. 58, 2325 (1987). 
[37] Z. Rácz and M. Plischke, Phys. Rev. E 50, 3530 (1994).

[38] G. Wagner, P. Meakin, J. Feder, and T. Jossang, Phys. Rev. E 55, 1698 (1997); see page 1702.

[39] M. Avellaneda, F. Elliott, and C. Apelian, J. Stat. Phys. 72, 1227 (1993).

[40] P. Meakin, P. Ramanlal, L. M. Sander, and R. C. Ball, Phys. Rev. A 34, 5091 (1986).

[41] M. Plischke, Z. Rácz, and D. Liu, Phys. Rev. B 35, 3485 (1987).

[42] D. Liu and M. Plischke, Phys. Rev. B 38, 4781 (1988).

[43] In retrospect, the Kim-Kosterlitz model (J. M. Kim and J. M. Kosterlitz, Phys. Rev. Lett. 62, 2289 (1989)) probably provides a faster crossover to the asymptotic behavior than the single-step model.

[44] This makes the algorithm nonlocal, since the rate at which deposition occurs on an allowed site depends on the number of such sites in the rest of the system.

[45] H. van Beijeren, Phys. Rev. Lett. 38, 993 (1977).

[46] M. Lässig, Phys. Rev. Lett. 80, 2366 (1998).

[47] F. Family, in Dynamical Phenomena at Interfaces, Surfaces and Membranes, edited by D. Beysens, N. Boccara, and G. Forgacs (Nova Science, 1993).

[48] T. Ala-Nissila, T. Hjelt, J. M. Kosterlitz, and O. Venalainen, J. Stat. Phys. 72, 207 (1993); L.-H. Tang, B. M. Forrest, and D. E. Wolf, Phys. Rev. A 45, 7162 (1992); C.-C. Chin and M. den Nijs, Phys. Rev. E 59, 2633 (1999).

[49] G. Palasantzas and J. Krim, Phys. Rev. Lett. 73, 3564 (1994).

[50] P. Kleban, J. Krim, and C. Ruffing, in Proc. Symposium on "Structure and Evolution of Surfaces", Ed. Cammarata, R.C. Chason, E.H. Einstein, T.L. Williams (MRS Meeting, Boston, 1996).

[51] J. M. Gómez-Rodŕiguez, A. M. Baró, and R. C. Salvarezza, J. Vac. Sci. Technol. B 9, 495 (1991).

[52] Z. Csahok, Z. Farkas, M. Menyhard, G. Gergely, and C. S. Daroczi, Surface Science 364, L600 (1996).

[53] H.-N. Yang and T.-M. Lu, Phys. Rev. B 51, 2479 (1995).

[54] W. M. Tong, R. S. Williams, A. Yanase, Y. Segawa, and M. S. Anderson, Phys. Rev. Lett. 72, 3374 (1994).

[55] The ensemble distribution functions (for many independent samples) of the system-wide height variance is another characterization of roughness; see Ref. [37].

[56] P. Constantin, I. Procaccia, and K.R. Sreenivasan, Phys. Rev. Lett. 67, 1739 (1991).

[57] H.J. Catrakis and P.E. Dimotakis, Phys. Rev. Lett. 80, 968 (1998).

[58] J. Kondev and G. Huber, unpublished.

[59] J. Kondev and C. L. Henley, Phys. Rev. B 52, 6628 (1995).

[60] J. Kondev and C. L. Henley, Nuc. Phys. B464, 540 (1996).

[61] M.T. Batchelor, B. Nienhuis, and S.O. Warnaar, Phys. Rev. Lett. 62, 2425 (1989).

[62] M.T. Batchelor, J. Suzuki, and C.M. Yung, Phys. Rev. Lett. 73, 2646 (1994).

[63] S. A. Trugman, Phys. Rev. B 27, 7539 (1983).

[64] M. P. M. den Nijs, J. Phys. A 12, 1857 (1979). 
TABLE I. Geometrical exponents $x_{l}, D_{f}$, and $\tau$ for loops on Gaussian surfaces with various roughness exponents $\alpha$. Columns marked "direct" are from direct fits to a power law of the data from system size $L=512$, inferring $2 x_{l}, D_{f}$, and $\tau-2$ from plots

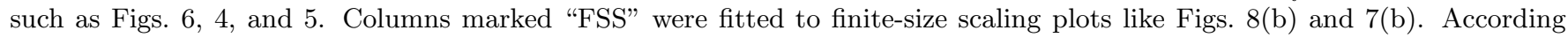
to our conjecture, the "theory" value of $2 x_{l}$ is 1 , independent of $\alpha$, and this is supported by the measurements here. Notice a slight systematic deviation of the "direct" exponents from theory when $\alpha>0.5$, which we attribute to more severe finite size effects in those cases. The "theory" formulas for $D_{f}$ and $\tau-2$ are in Eq. (4.13).

\begin{tabular}{|c|c|c|c|c|c|c|c|c|}
\hline$\alpha$ & \multicolumn{2}{|c|}{$2 x_{l}$} & \multicolumn{3}{|c|}{$D_{f}$} & \multicolumn{3}{|c|}{$\tau-2$} \\
\hline & direct & $\overline{F S S}$ & direct & FSS & theory & direct & FSS & theory \\
\hline 0.0 & $1.07(2)$ & $1.02(2)$ & $1.48(1)$ & $1.50(2)$ & 1.5 & $0.35(2)$ & $0.33(1)$ & $0.333 \ldots$ \\
\hline 0.2 & $1.04(1)$ & $0.97(2)$ & $1.39(1)$ & $1.41(2)$ & 1.4 & $0.30(1)$ & $0.28(1)$ & $0.286 \ldots$ \\
\hline 0.4 & $1.01(1)$ & $0.98(2)$ & $1.31(2)$ & $1.32(3)$ & 1.3 & $0.24(1)$ & $0.225(5)$ & $0.231 \ldots$ \\
\hline 0.6 & $1.00(1)$ & $0.97(2)$ & $1.23(3)$ & $1.19(3)$ & 1.2 & $0.18(1)$ & $0.165(5)$ & $0.166 \ldots$ \\
\hline 0.8 & $0.97(2)$ & $0.97(2)$ & $1.15(1)$ & $1.11(2)$ & 1.1 & $0.12(1)$ & $0.11(2)$ & $0.090 \ldots$ \\
\hline
\end{tabular}

TABLE II. Results of fits to roughness measures applied to surfaces generated by the single-step model. The exponent values $\alpha$ in the left two columns were derived in two direct ways, from the data plotted in Figures 12 (a) and 11. The direct-fit results ("direct") for $D_{f}$ and $\tau-2$ used only the $L=128$ data (shown in Figures 13 and 14); the finite-size scaling results ("FSS") were obtained using system sizes $L=32,64,128$. The subheadings " $\alpha$ " under $D_{f}$ and $\tau-2$ are estimates of the roughness exponent obtained from the FSS results by inverting Eq. (4.13).

\begin{tabular}{|c|c|c|c|c|c|c|c|c|}
\hline \multirow[t]{2}{*}{$\underline{p_{+}}$} & \multicolumn{2}{|c|}{$\alpha$} & \multicolumn{3}{|c|}{$D_{f}$} & \multicolumn{3}{|c|}{$\tau-2$} \\
\hline & $\left\langle C_{b}^{2}\right\rangle$ & $\left\langle|\tilde{h}(\mathbf{q})|^{2}\right\rangle$ & direct & FSS & $\alpha$ & direct & FSS & $\alpha$ \\
\hline$\overline{0.1}$ & $0.33(2)$ & $0.35(1)$ & $1.38(1)$ & $1.35(2)$ & $\overline{0.30(4)}$ & $0.30(1)$ & $0.24(1)$ & $0.37(3)$ \\
\hline 0.3 & $0.19(4)$ & $0.09(2)$ & $1.47(1)$ & $1.46(2)$ & $0.08(4)$ & $0.38(1)$ & $0.35(1)$ & $-0.08(5)$ \\
\hline 0.5 & $0.135(2)$ & 0.08 (1) & $1.51(2)$ & $1.50(2)$ & $0.00(4)$ & $0.40(2)$ & $0.36(2)$ & $-0.13(5)$ \\
\hline
\end{tabular}

TABLE III. Roughness measures

\begin{tabular}{ll}
\hline \hline Quantity & Description \\
\hline Quadratic measures & \\
\hline 1. $\left\langle\left(h(\mathbf{x})-\bar{h}_{b}\right)^{2}\right\rangle_{b}$ & variance in $b \times b$ patch \\
2. $D_{2}(\mathbf{r})=\left\langle|h(\mathbf{r})-h(0)|^{2}\right\rangle$ & height correlation \\
3. $\left\langle|\tilde{h}(\mathbf{q})|^{2}\right\rangle$ & Fourier spectrum \\
4. $\left\langle\left|C_{b}(\mathbf{x})\right|^{2}\right\rangle$ & " $b$-dependent curvature" variance \\
\hline Cubic and other non-quadratic measures & \\
\hline $5 .\left\langle\left(h(\mathbf{r})-\bar{h}_{b}\right)^{3}\right\rangle_{b}$ & skew moment in $b \times b$ patch \\
6. \langle|$\left(h(\mathbf{r})-\left.h(0)\right|^{q}\right\rangle$ & $q$-multiaffine height correlation \\
7. $\left\langle\left(H_{b}(\mathbf{r})-\bar{h}\right)^{3}\right\rangle$ & skew moment of $b$-smoothed height \\
8. $\left\langle\left[C_{b}(\mathbf{x})\right]^{3}\right\rangle$ & "curvature" skew moment \\
9. $\left\langle\left[C_{b}(\mathbf{x})\right]^{4}\right\rangle$ & "curvature" quartic moment \\
\hline Loop measures & \\
\hline $10 .\langle s\rangle_{R}$ & average loop length, given radius $R$ \\
$11 . \quad P_{>}(s)$ & Prob (loop through $\mathbf{x}$ is longer than $s)$ \\
$12 . G(\mathbf{r})$ & loop connectedness correlation function \\
\hline \hline
\end{tabular}




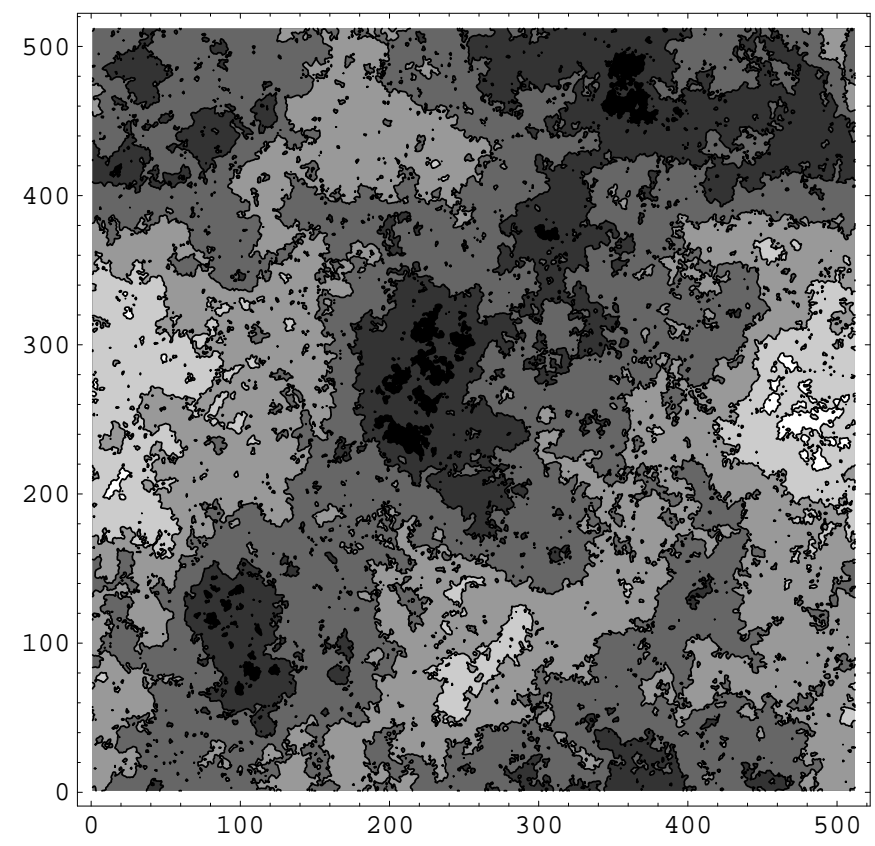

FIG. 1. Contour plot of a $\alpha=0.4$ random Gaussian surface; system size $\mathrm{L}=512$. 

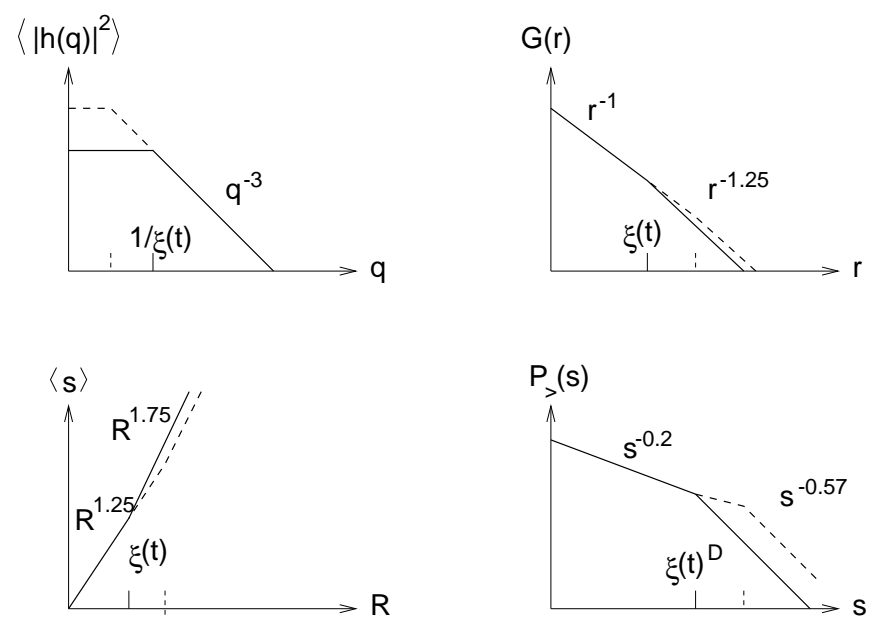

FIG. 2. Percolation crossover. In each figure, the solid line represents the function at a certain time $t$, and the dashed line represented the same function at a later time when $\xi(t)$ has increased. The exponents shown in the figures are for $\alpha=1 / 2$, but the qualitative behavior is the same so long as $0<\alpha<1$. Each graph shows a crossover to percolation exponents at a "knee" which corresponds to a length scale $\sim \xi(t)$ : (a) Fourier spectrum $\left\langle|\tilde{h}(\mathbf{q})|^{2}\right\rangle$, (b) loop correlation function $G(r)$, (c) average loop length $\langle s\rangle(R)$, and (d) cumulative distribution $P_{>}(s)$ of loop lengths (through a given point).
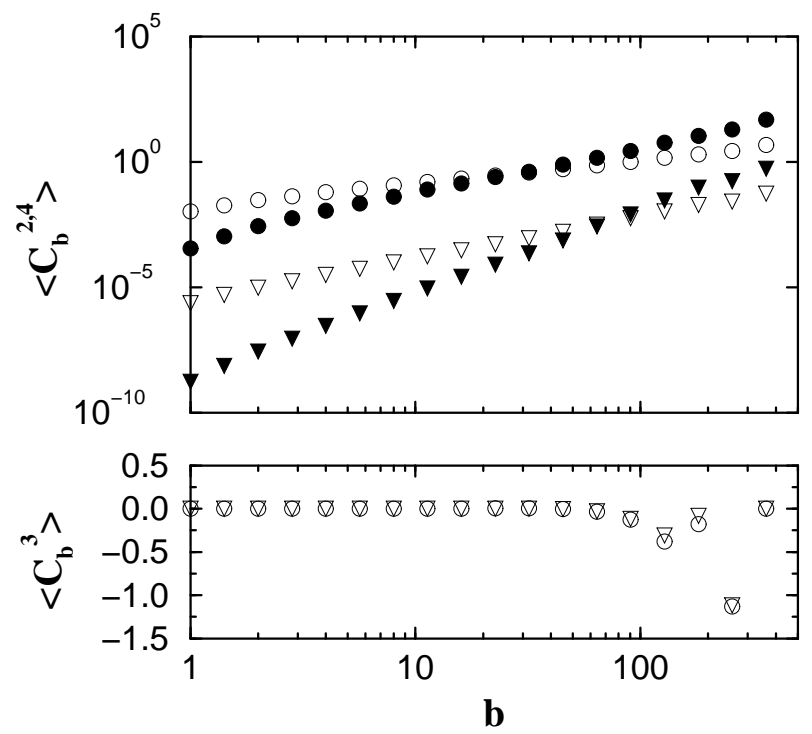

FIG. 3. Scale-dependent curvature moments from Gaussian random surfaces with roughness exponents $\alpha=0.4$ (circles) and 0.8 (triangles). The third moments (lower plot) are zero confirming the up/down symmetry. The upper plot shows the second moments (open symbols) and fourth moments (filled symbols). 


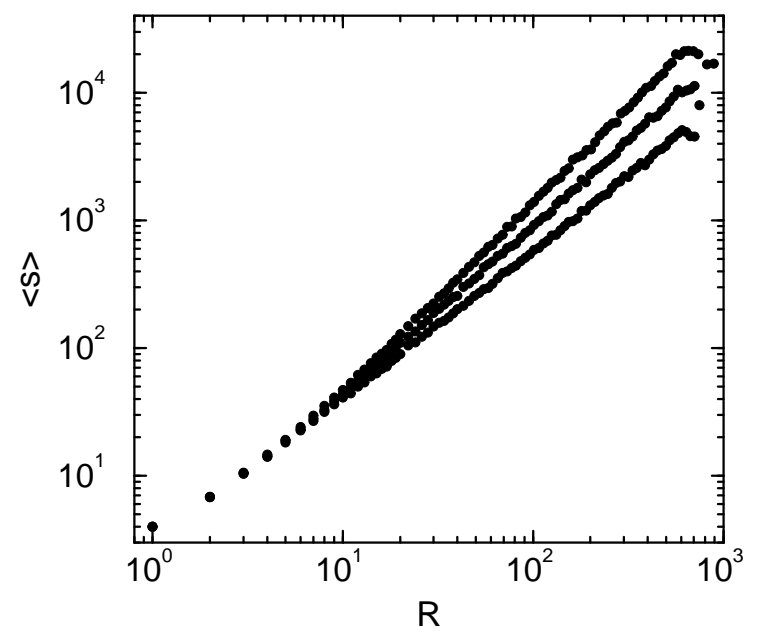

FIG. 4. Average loop length $\langle s\rangle$ as a function of loop radius $R$, for random Gaussian surfaces with $\alpha=0,0.4,0.8$ (from top to bottom); system size $L=512$, and $10^{4}$ loops were collected. The "direct" $D_{f}$ data in Table I are obtained by linear least-squares fits to such plots in the scaling regime, which is roughly $10<R<100$.

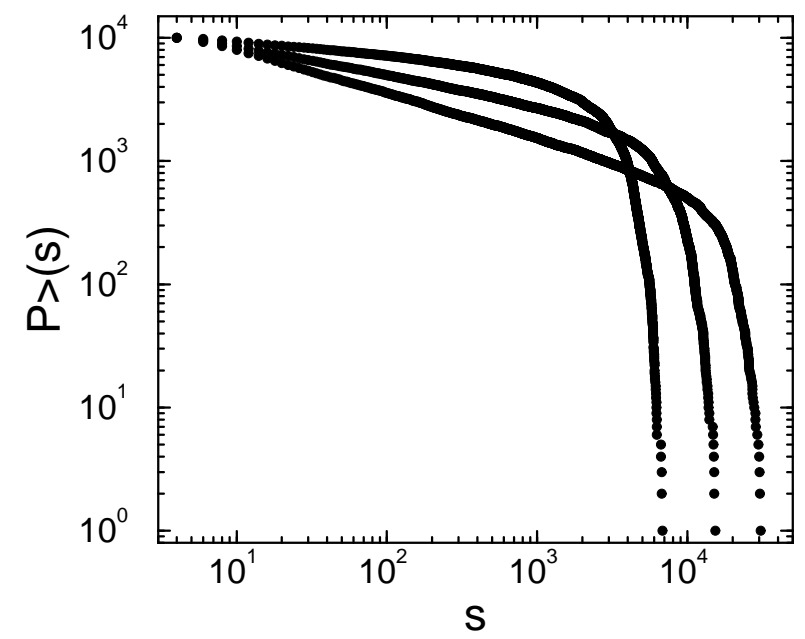

FIG. 5. Cumulative number of loops whose length is bigger than $s$ for random Gaussian surfaces with $\alpha=0,0.4,0.8$ (from top to bottom); system size $L=512$. Here and in all other plots of $P_{>}(s)$, raw data is binned in intervals of form $(s, 1.1 s)$. 


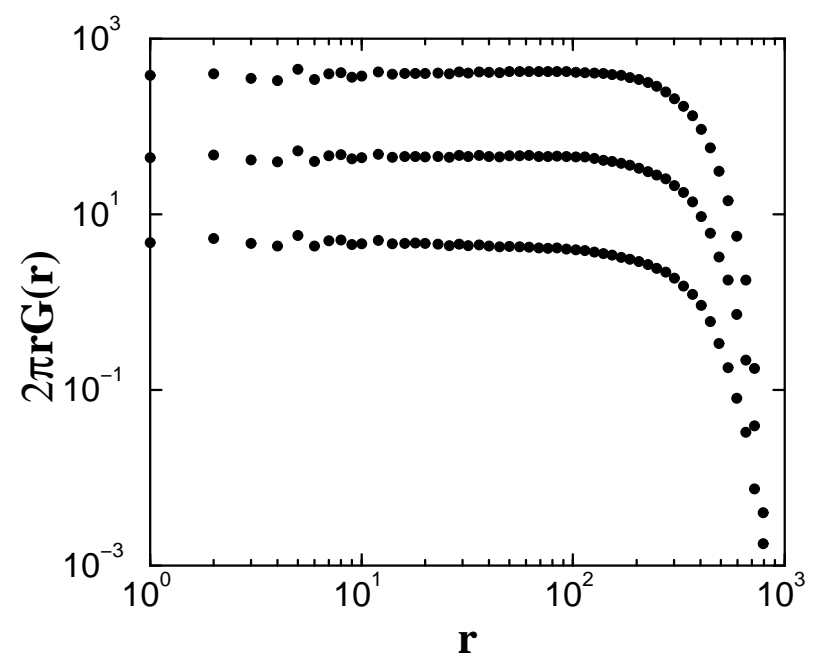

FIG. 6. Loop correlation function for random Gaussian surfaces with $\alpha=0,0.4,0.8$ (from bottom to top); system size $L=512$. In this and all such plots, raw data is binned logarithmically in intervals of form $(r, 1.1 r)$. The latter two graphs are offset vertically by factors of 10 for clarity; they are virtually identical except for a "knee" at slightly different $r$ values. 

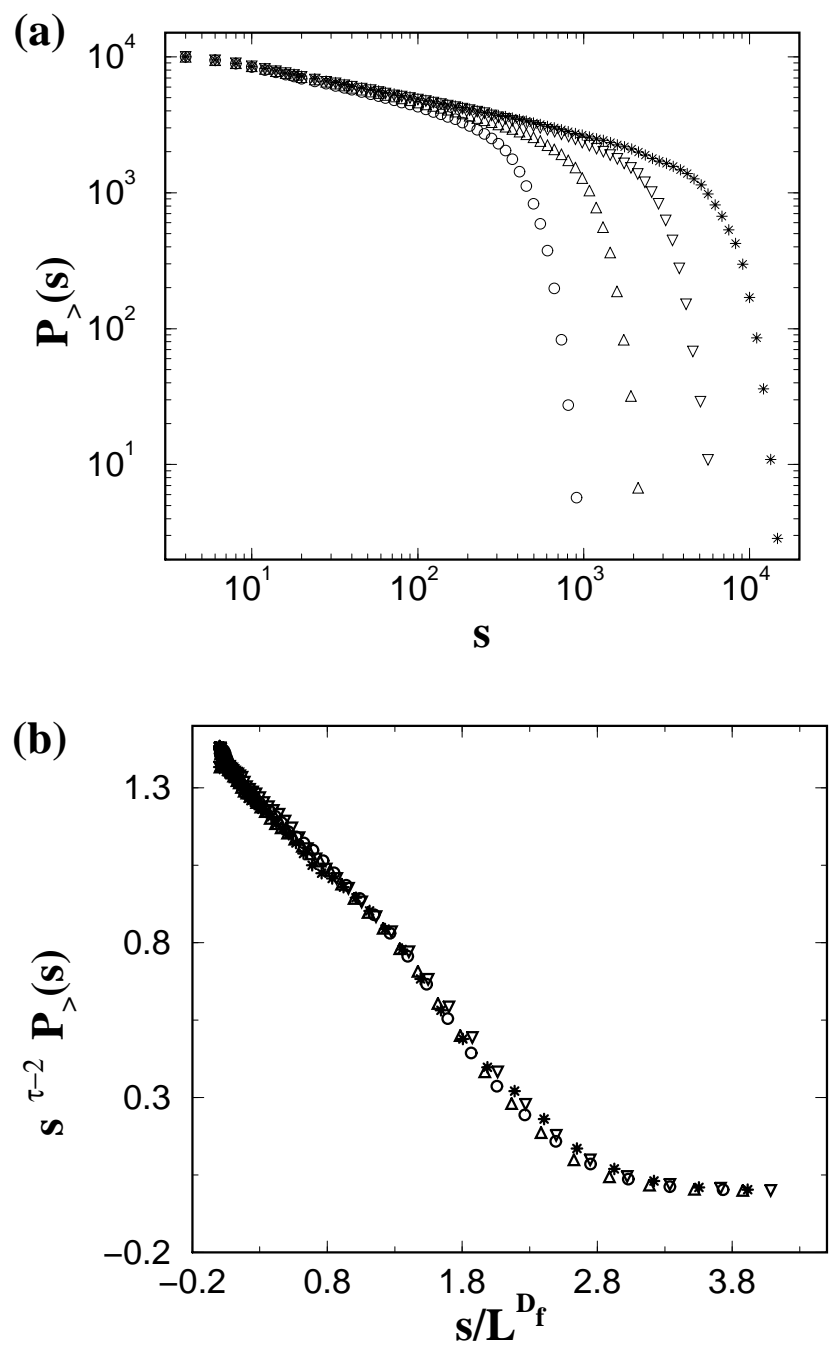

FIG. 7. Cumulative loop-size distribution, for random Gaussian surfaces with $\alpha=0.4$. (a) Data for system sizes $L=64(\bigcirc), L=128(\triangle) L=256(\nabla)$, and $L=512$ $(*)$. (b) Collapse of this data in a finite-size scaling plot with $\tau-2=0.225$ and $D_{f}=1.32$. 

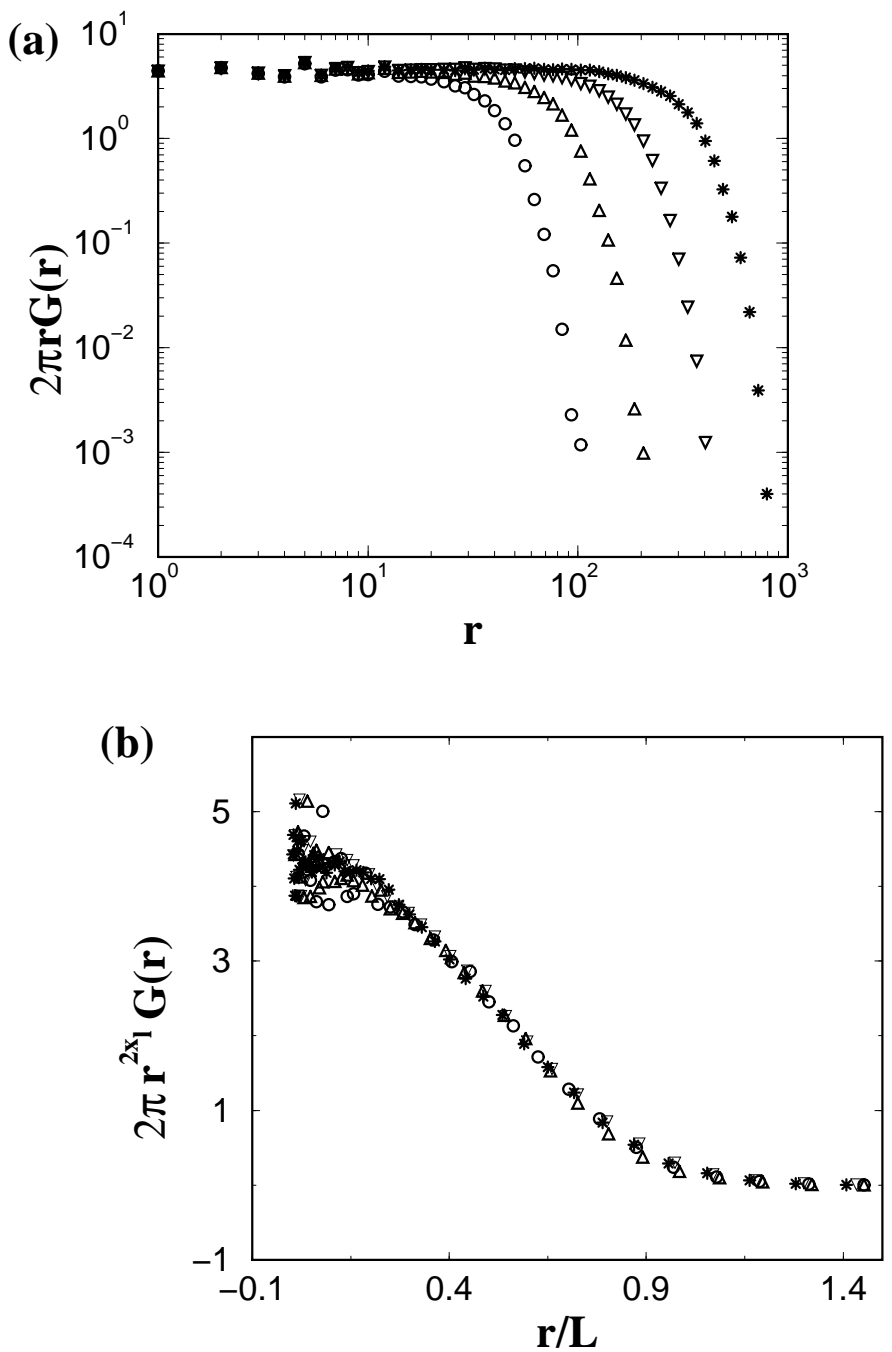

FIG. 8. System-size dependence of the loop correlation function $G(r)$ for Gaussian random surfaces with $\alpha=0.4$. (a) Data for sizes $L=64(\bigcirc), L=128(\nabla) L=256(\nabla)$, and $L=512(*)$. (b) Data collapse of this data with $2 x_{l}=1.02$. 


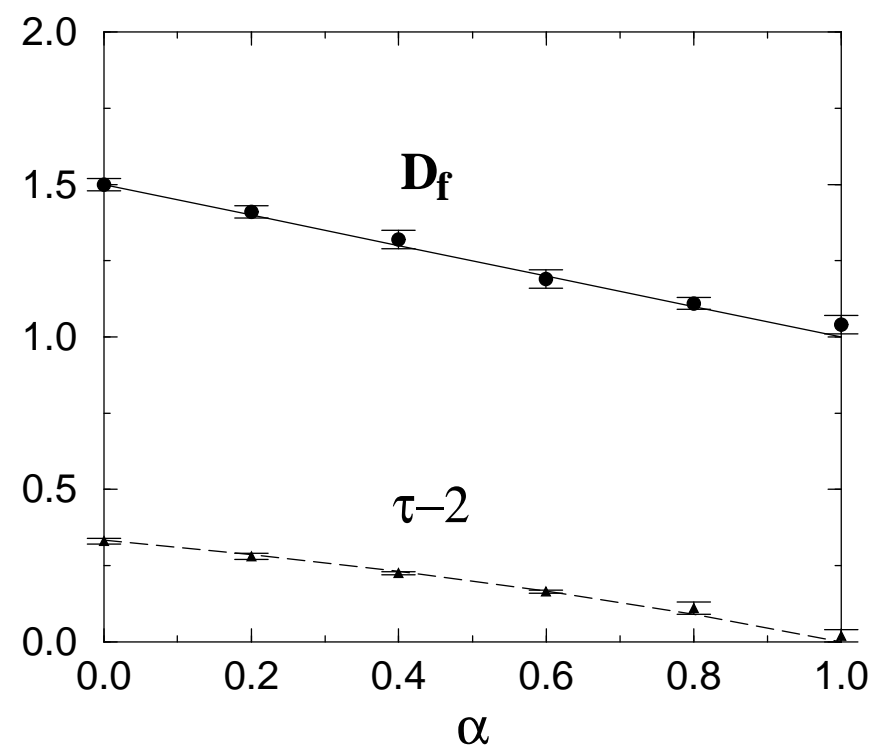

FIG. 9. Fractal dimension $D_{f}$ and length distribution exponent $\tau-2$, as functions of the roughness exponent $\alpha$ of a random Gaussian surface, obtained from finite-size scaling fits (see Table $\mathbb{1}$ ). The solid and dashed lines corresponds to the formulas in Eq. (4.13).
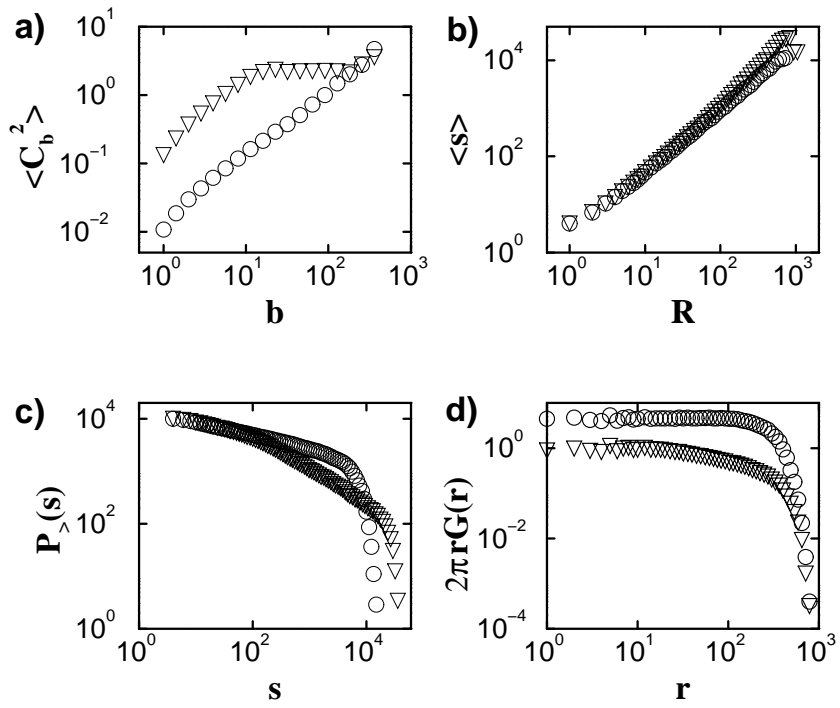

FIG. 10. Finite correlation length effects for Gaussian random surfaces with $\alpha=0.4$, and a crossover to white noise for wavevectors smaller than $\pi / \xi_{q}$, where $\xi_{q}=16 ; L=512$ is the system size. Circles are used for data sets with no cutoff which are included here for comparison with the cutoff data (triangles). a) Squared curvature function - note the knee at $b \approx 15$. b) Average loop length as function of radius - knee at $R \approx 20$. c) Cumulative distribution of loop sizes - knee at $s \approx 100$. d) Loop correlation function - knee at $r \approx 20$. 


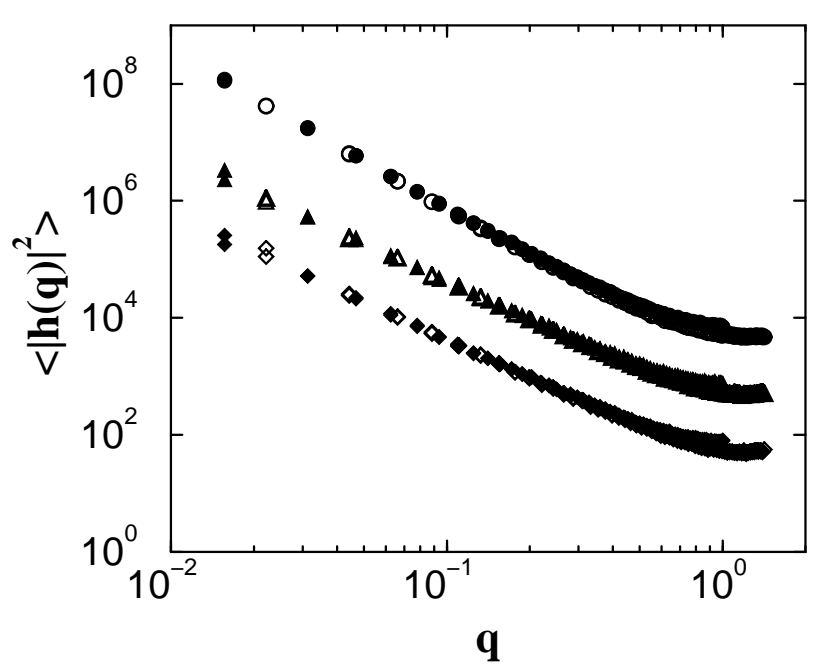

FIG. 11. Power spectrum of the height in the SSM model along the $[1,0]$ (filled symbols) and the $[1,1]$ direction (open symbols) in reciprocal space. The data for $p=0.3$ (triangles) and for $p_{+}=0.5$ (diamonds) has been shifted with respect to the $p_{+}=0.1$ data (circles) by factors of 0.1 and 0.01 respectively (for clarity). Note that the power spectrum is isotropic in Fourier space for small values of $|\mathbf{q}|$. 

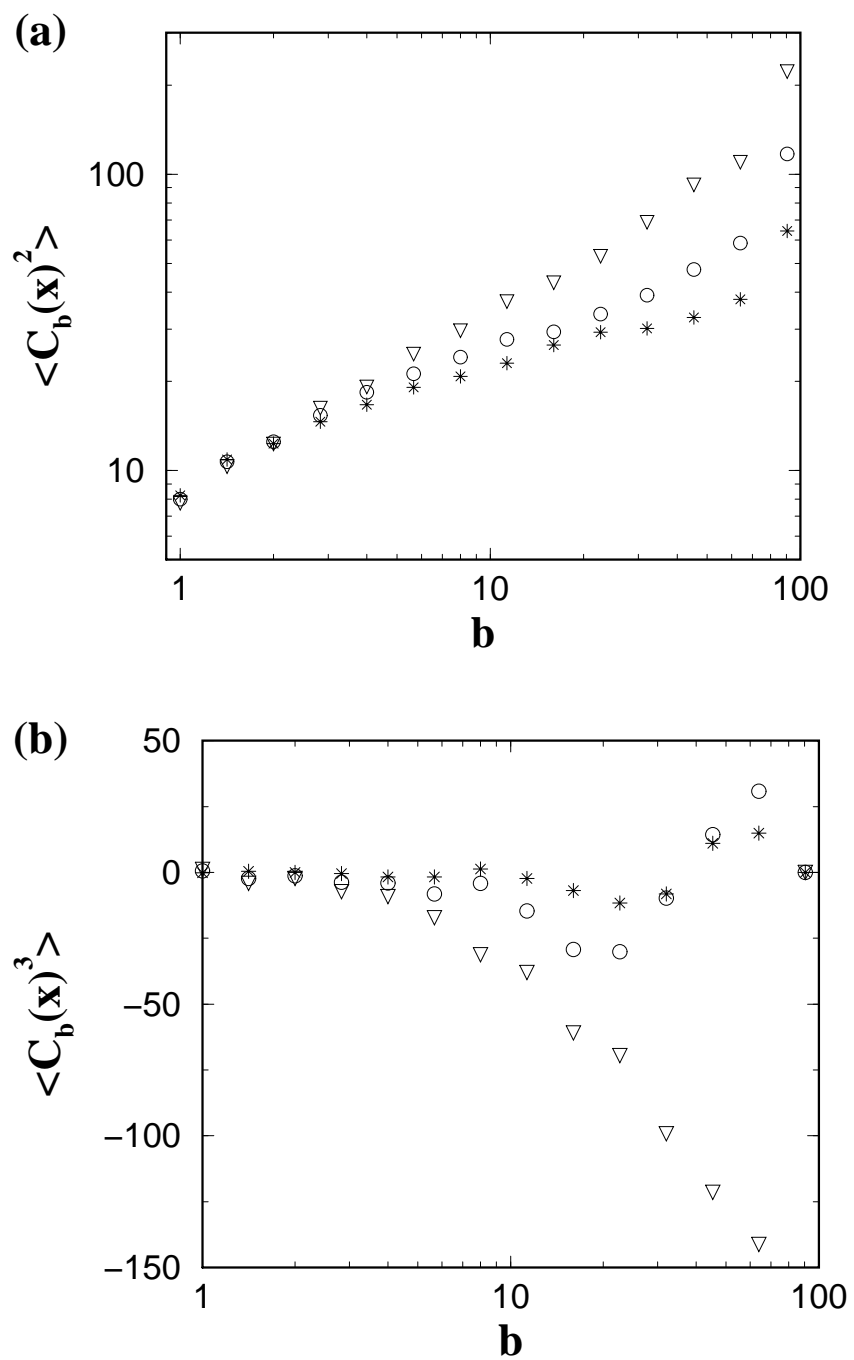

FIG. 12. Second moment (a) and third moment (b) of the scale-dependent curvature $C_{b}(\mathbf{x})$, for surfaces from the single-step model with $p_{+}=0.1(\triangle), p_{+}=0.3$ (o), and $p_{+}=0.5(*)$. In (b), the $p_{+}=0.3$ and 0.5 data are consistent with $\left\langle C_{b}^{3}\right\rangle=0$, while the $p_{+}=0.1$ data show a strong (and non-Gaussian) breaking of up/down symmetry. 


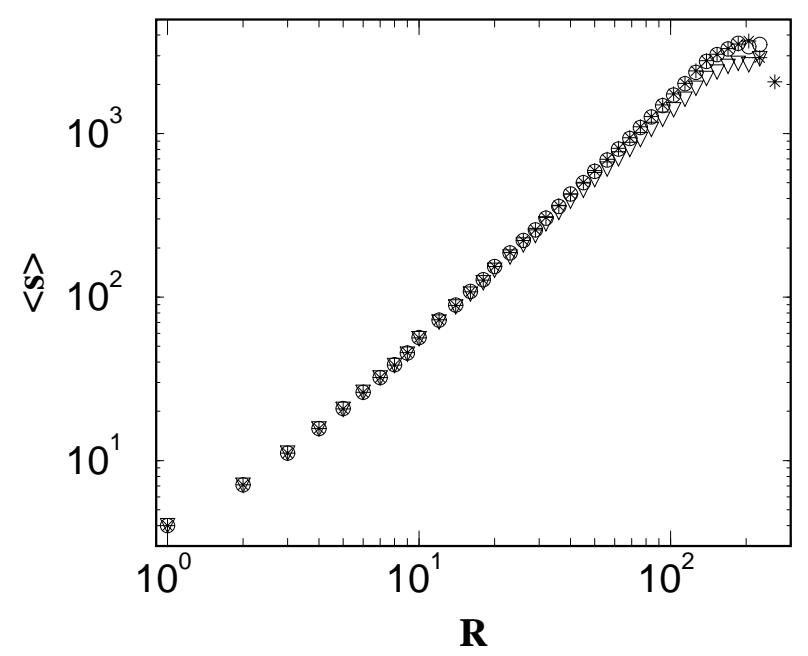

FIG. 13. Average loop length "vs" the loop radius for $p_{+}=0.1(\triangle), p_{+}=0.3(\circ)$, and $p_{+}=0.5(*)$, in the single-step model. Note that the $p_{+}=0.3$ and $p_{+}=0.5$ data are almost indistinguishable.

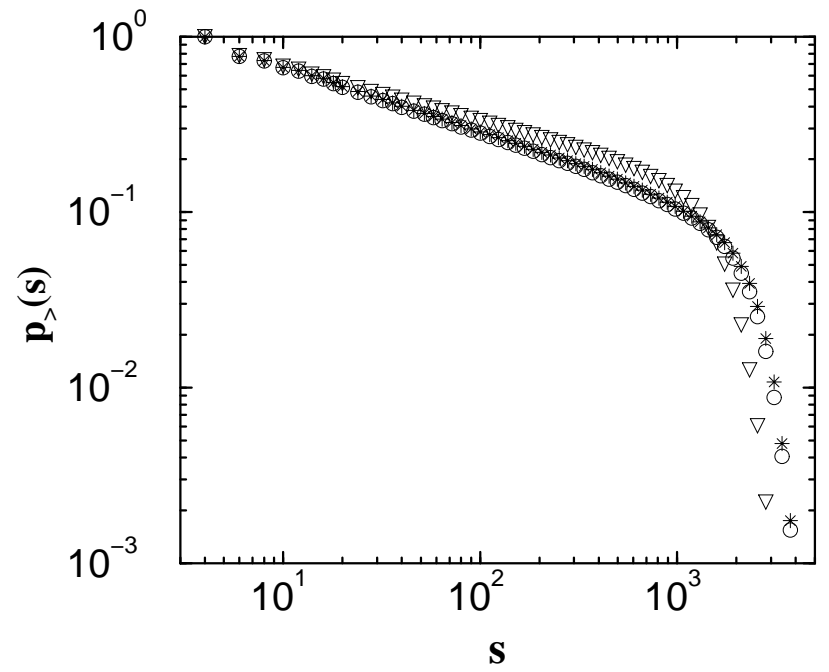

FIG. 14. Normalized loop size distribution for $p_{+}=0.1$ $(\triangle), p_{+}=0.3(\circ)$, and $p_{+}=0.5(*)$, in the single step model. Again, the $p_{+}=0.3$ and $p_{+}=0.5$ plots are almost indistinguishable. 

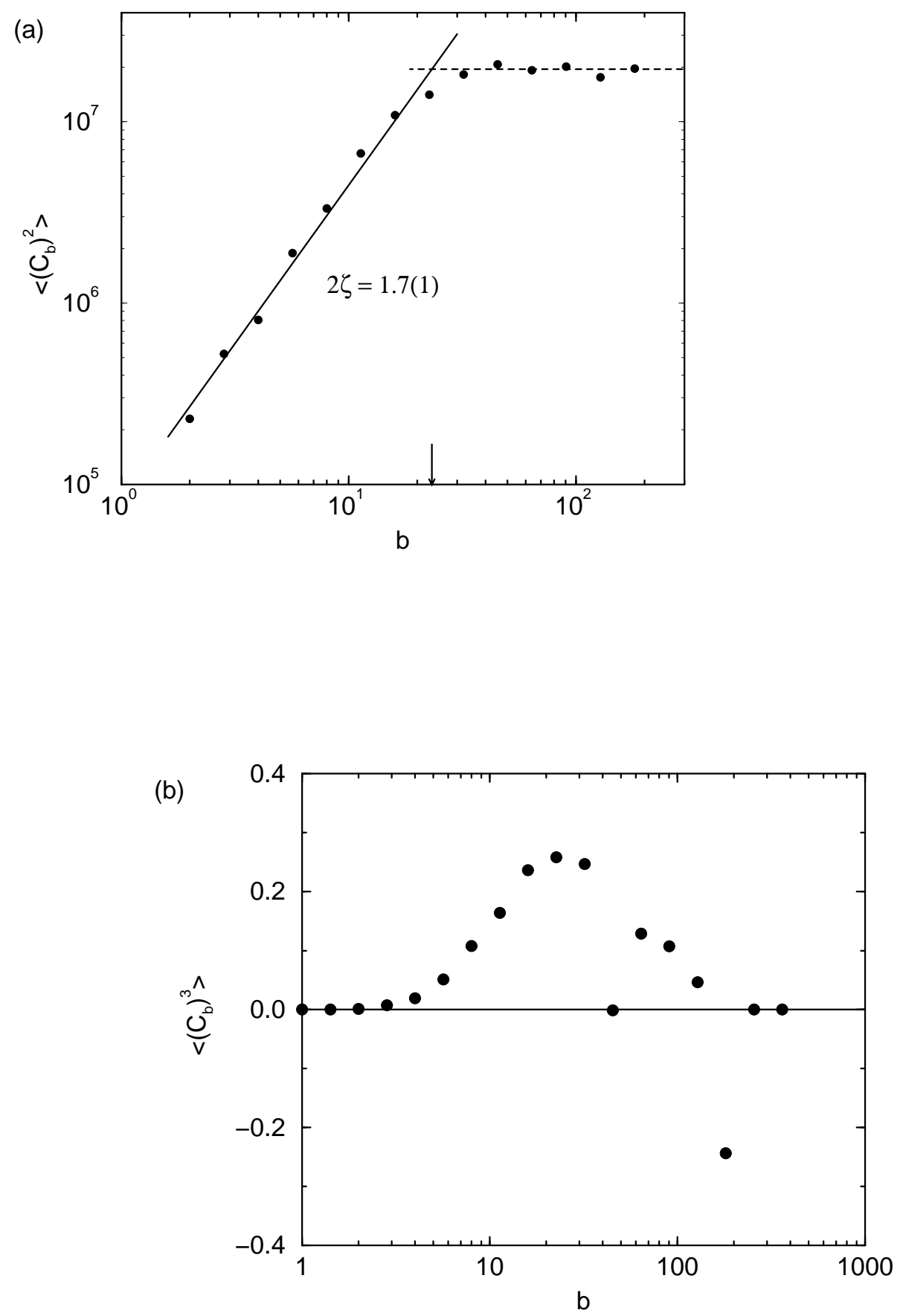

FIG. 15. Second moment (a) and third moment (b) of the scale-dependent curvature, as evaluated for a $702 \mathrm{~nm}$ thick Ag film, grown on quartz, from the STM data of Palasantzas and Krim (Ref. 449). 


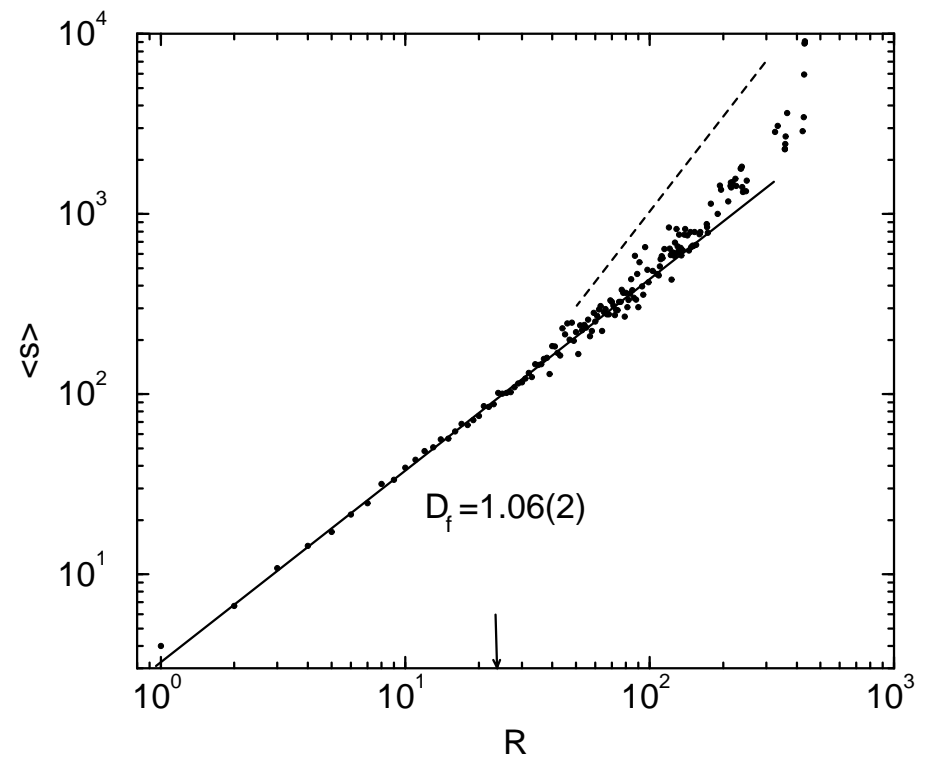

FIG. 16. Mean contour length $\langle s\rangle$ as a function of radius $R$, for the Ag film of Ref. 49. Here 1000 contour loops were collected from the STM data of Ref. [49]. The solid line is the least-squares best fit for radii $2<R<125$; its slope is the estimated fractal dimension $D_{f}$. The slope of the dashed line is equal to the hull dimension of critical percolation clusters. 


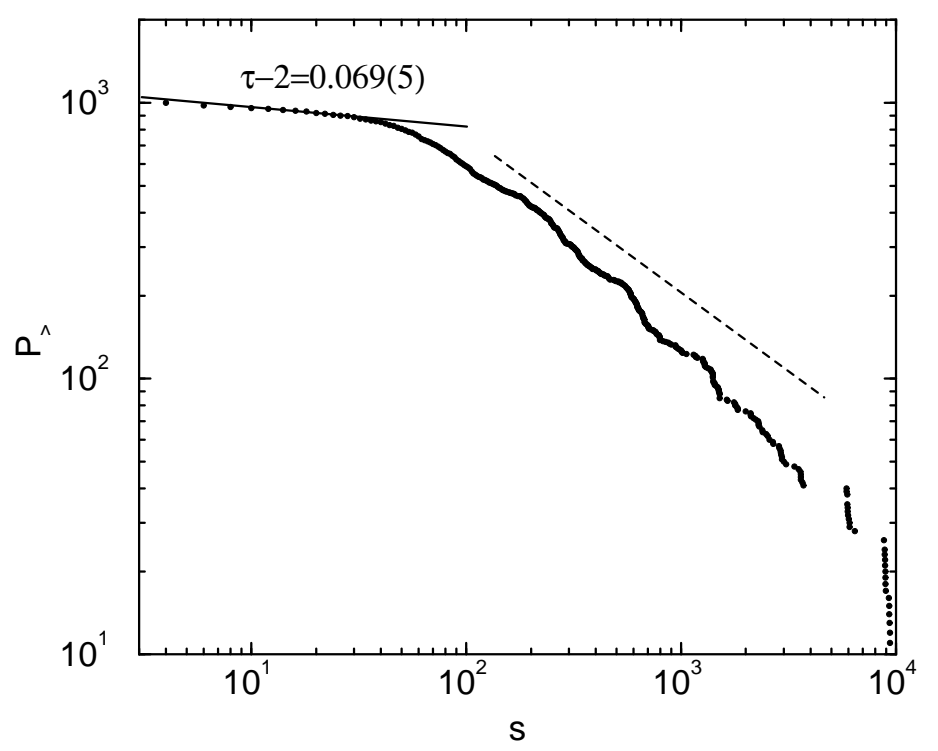

FIG. 17. Cumulative distribution of contour loop lengths from STM data, for the Ag film of Ref. 49. The solid line is the result of a linear fit to the data in the affine-scaling regime. The slope of the dashed line corresponds to the exponent $\tau-2$ in the percolation regime. 


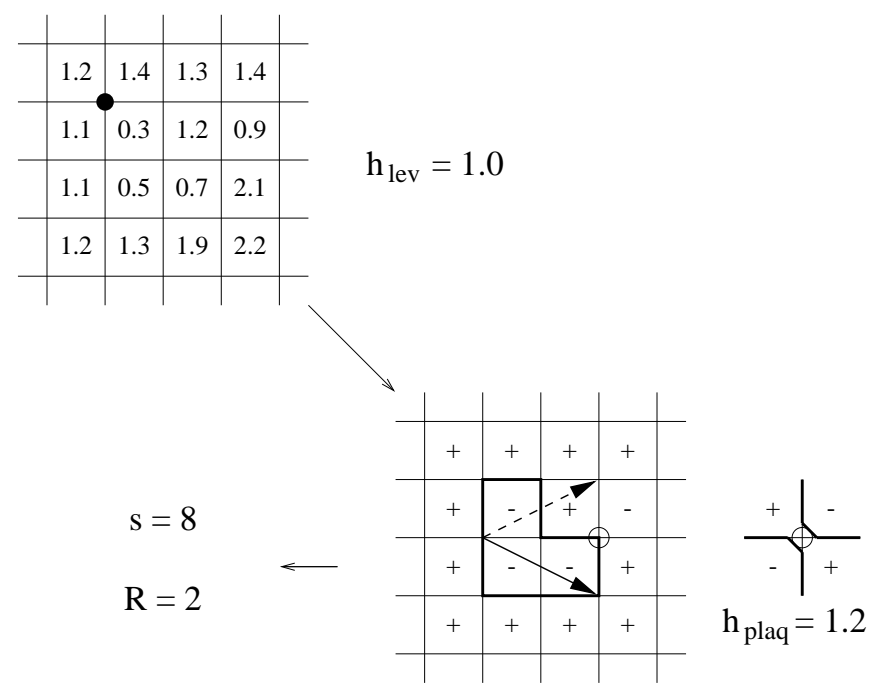

FIG. 18. Construction of contour loops of a random surface on a lattice. Heights $h(\mathbf{r})$ are indicated by numbers in the cells; $h_{\text {lev }}$ is the height of the level set through the chosen point (filled circle) while $h_{\text {plaq }}$ is the height of the "saddle-point" (unfilled circle). Our definition of the diameter $R$ and loop length $s$ is indicated. The solid arrow connects points on the same loop, and thus contributes to the loop correlation function $G(\mathbf{r})$; the dashed arrow does not contribute: it connects points of the same level set, but they are on disconnected loops. 